\title{
Approximate global convergence and quasi-reversibility for a coefficient inverse problem with backscattering data
}

\author{
Andrey V. Kuzhuget* Larisa Beilina ${ }^{\dagger} \quad$ Michael V. Klibanov* \\ and Vladimir G. Romanov $\stackrel{\ddagger}{+}$
}

\begin{abstract}
A numerical method with the approximate global convergence property is developed for a 3-D Coefficient Inverse Problem for a hyperbolic PDE with the backscattering data. An important part of this technique is the quasi-reversibility method. Approximate global convergence theorem is proven. Results of two numerical experiments are presented.
\end{abstract}

KEY WORDS: coefficient inverse problem, backscattering data, approximate global convergence, quasi-reversibility method, numerical study

AMS subject classification: 15A15, 15A09, 15A23

This preprint is available on-line at http://www.ma.utexas.edu/mp_arc/index-10.html.

\section{Introduction}

The goal of this publication is to extend the numerical method of $[4,5,6,7,8,27]$ to the case of backscattering data resulting from a single measurement. "Single measurement" means that the time dependent backscattering data for a Coefficient Inverse Problem (CIP) for a hyperbolic PDE are generated by a single location of the point source. We develop a new theory and present numerical results confirming this theory. Our target application is in imaging of plastic antipersonnel land mines, and we model this application in our numerical testing. In military applications, due to various dangers on the battlefield, it is desirable to minimize both the number of measurements and the observation angle. In the current paper

\footnotetext{
*Department of Mathematics and Statistics, University of North Carolina at Charlotte, Charlotte, NC 28223, USA (akuzhuge@uncc.edu, mklibanv@uncc.edu).

${ }^{\dagger}$ Department of Mathematical Sciences, Chalmers University of Technology and Gothenburg University, SE-42196 Gothenburg, Sweden, (larisa@chalmers.se).

${ }^{\ddagger}$ Sobolev Mathematical Institute of the Siberian Branch of the Russian Academy of Sciences, Koptyug Prospect 4, Novosibirsk 630090, Russia (romanov@math.nsc.ru).
} 
we model the most suitable arrangement for this case, which is to use a single position of the point source and to measure only the backscattering signal.

It will be shown in the forthcoming publication [31] that a close analog of the numerical method of this paper provides accurate results for the most challenging case of blind backscattering experimental data collected from targets mimicking antipersonnel land mines. These data were collected in the field by a new forward looking radar, see [36] for a description of this radar.

This is a significantly revised version of the paper [30]. Main analytical results of the current paper: Theorems 3.1, 6.1, 7.1, Lemma 7.1 and topics of subsections 6.2 and 6.3 are not parts of [30]. Lemmata 5.1, 5.2 were formulated but not proven in [30]. Lemma 5.3 was proven in [30]. Theorem 5.1 was proven in [30] for the 2-D case. Since the proof in 3-D is similar, we omit it here. Numerical examples of this paper as well as Figures 8.1,8.2 are different from ones of [30].

\subsection{Preliminaries}

It is well known that the goal of the construction of reliable numerical methods for CIPs faces quite substantial challenges. They are mainly caused by two factors combined: nonlinearity and ill-posedness of CIPs. Least squares regularizing Tikhonov functionals suffer from the commonly known phenomenon of multiple local minima and ravines. Therefore, a gradient-like method of the minimization of this functional can stop at any point of either a local minimum or a ravine. However, this point can be located quite far from the true solution. This results in the local convergence of conventional numerical methods for CIPs, such as, e.g. various versions of the Newton and gradient methods. Convergence of these algorithms is guaranteed only if the starting point of iterations is located in a sufficiently small neighborhood of the exact solution. At the same time, in many applications a good approximation for the exact solution is rarely known in advance.

Therefore the central question in a computational treatment of a CIP is to develop such a numerical method, which would provide at least one point in a sufficiently small neighborhood of the exact solution without any a priori knowledge of that neighborhood. Furthermore, the latter property should be rigorously guaranteed, at least within the framework of a certain reasonable approximate mathematical model. In addition, numerical studies should confirm this property.

This question was addressed in a series of recent publications $[4,5,6,7,8,27]$ for CIPs for a hyperbolic PDE in the case when the data resulting from a single measurement are given at the entire boundary, i.e. the case of complete data collection. The technique of neither these publications nor the current one does not use least squares functionals. Only the structure of the underlying PDE operator is used. Also, it does not use a knowledge of the background values of the unknown coefficient.

Because of a quite significant difficulty of addressing the above question, it is unlikely that this can be done without some approximations. Hence, a reasonable approximate mathematical model was proposed on pages 102-104 of [5] and on page 11 of [27]. Developing this 
concept further, we introduce in subsection 6.2 a new concept of the so-called approximate global convergence property. Based on this concept, we specify in subsection 6.3 our new approximate mathematical model.

As soon as the desired first good approximation is obtained, a locally convergent numerical method can be applied to refine it, see, e.g. books [2, 24] for Newton-like methods for ill-posed problems. Thus, a two-stage numerical procedure was developed in $[5,6,7,8,30]$, and it also takes place here. On the first stage a good first approximation is provided by the above technique. On the second stage this approximation is refined via a locally convergent method. This method takes the first stage solution as the starting point for iterations.

In addition to the synthetic data of numerical studies of $[4,5,6,7]$, an experimental verification of the first stage was carried out in [27] for the case of blind experimental data collected in the transmitted scattering mode. A very accurate reconstruction of both refractive indices and locations of dielectric inclusions was demonstrated in [27]. Next, the two-stage procedure was verified on the same experimental data in [8]. A very good reconstruction accuracy of locations, shapes and refractive indices of dielectric inclusions was observed in [8].

\subsection{What is new in this paper}

Since we have over-determined boundary conditions on the backscattering part of the boundary of the domain of interest, we use the Quasi-Reversibility Method (QRM) [28, 33]. The QRM is well suited for this kind of problems, since it finds the "least squares" solution. The QRM was not in $[4,5,6,7,8,27]$ since the complete data collection was used in these references. We refer to, e.g. [14, 15, 16, 18, 25] for some publications on the QRM for linear ill-posed problems. The convergence analysis of the QRM is based on Carleman estimates.

The goal of the analytical part of this paper is to establish the above mentioned approximate global convergence property for our algorithm. While the QRM is well known for linear ill-posed problems, its application to a nonlinear problem, such as our CIP, is new. The only exception in this regard is the recently published work [29] of the first and third authors, where the technique of $[4,5]$ was extended to the 1-D case with the backscattering data and the QRM was applied. However, the Carleman estimate in 1-D is simpler than the one in $n-\mathrm{D}(n=2,3)$ since in $1-\mathrm{D}$ it enables to estimate the $H^{2}$-norm of the solution in the whole interval of interest. Unlike this, the Carleman estimate in the $n-\mathrm{D}(n=2,3)$ case enables one to obtain the Hölder stability estimate of the $H^{2}$-norm of the QRM solution only in a subdomain of the domain of interest. This causes the first main difficulty in the convergence analysis compared with the linear case.

Let $\alpha \in(0,1)$ be a small regularization parameter of the QRM. The desired estimate of the $H^{2}$-norm of the solution in a subdomain inevitably contains the large multiplier $\alpha^{-r}, r=$ const. $>0$. This multiplier was not a problem in the linear case since the QRM is applied only once in this case. Now, however, because of the nonlinearity of our CIP, we need to arrange an iterative process. Thus, we need to "suppress" this multiplier on each iteration to ensure stability. This causes the second main difficulty, compared with the 
linear case. The third main difficulty here, compared with the linear case, is that along with the $H^{2}$-norm of the QRM solution in the subdomain, we need to iteratively estimate its $H^{5}$ - norm in the entire domain (because of the regularization term), and the latter estimate contains a large multiplier $\alpha^{-1 / 2}$ which again needs to be "suppressed" on each iteration to stabilize the process. Four more new analytical elements are:

1. To perform our convergence analysis, we establish in Theorem 3.1 (section 3) some new properties of the solution of an elliptic PDE in $\mathbb{R}^{3}$, including estimates of this solution both from the above and from the below. This PDE is obtained via the Laplace transform of the originating hyperbolic PDE. The difficulty here is that while the classical theory of elliptic PDEs is developed only for bounded domains [21], we need to work in $\mathbb{R}^{3}$.

2. We obtain accuracy estimates for the so-called "tail" functions on each iteration. Such estimates were not obtained in above cited previous publications about this method. These estimates lead to a more convenient (than previously) formulation of the approximate global convergence Theorem 7.1 .

3. We use a new Carleman Weight Function (CWF) in our Carleman estimate for the Laplace operator. This CWF is well suitable for a boundary condition, which is used here for a better stability of the QRM.

4. In the above cited previous publications about this method functions $q_{n, k}$ (section 4) were strong solutions of the Dirichlet boundary value problems for corresponding elliptic PDEs, and Schauder theorem was applied to estimate them. Now, however, each function $q_{n, k}$, is a weak solution of a certain integral identity since it is the minimizer of the Tikhonov functional corresponding to the QRM. This causes additional difficulties in the proof of Theorem 7.1.

While we work with the data resulting from a single measurement, we refer to works $[1,9,10,11,23,37]$ and references cited there for some non-locally convergent algorithms for CIPs with the data resulting from multiple measurements. In particular, publications $[9,10,23]$ consider CIPs for hyperbolic PDEs. In section 2 we pose the inverse problem. In section 3 we establish some properties of the Laplace transform of the solution of the forward problem. The algorithm is described in section 4. Sections 5-7 are about the convergence analysis. Numerical results are presented in section 8 .

\section{Posing the Inverse Problem}

Below $x=(x, y, z) \in \mathbb{R}^{3}$ denotes an arbitrary vector as well as the first component of this vector. The context does not allows an ambiguity. As the forward problem, we consider the Cauchy problem for the following hyperbolic PDE

$$
\begin{aligned}
c(x) u_{t t} & =\Delta u \text { in } \mathbb{R}^{3} \times(0, \infty), \\
u(x, 0) & =0, u_{t}(x, 0)=\delta\left(x-x_{0}\right) .
\end{aligned}
$$

Equation (1) governs, e.g. propagation of acoustic and electromagnetic waves. In the acoustical case $1 / \sqrt{c(x)}$ is the sound speed. In the $2-\mathrm{D}$ case of EM waves propagation in a 
non-magnetic medium $c(x):=\varepsilon_{r}(x)$, where $\varepsilon_{r}(x)$ is the spatially distributed dielectric constant, see, e.g. [17] for the derivation of (1) from the Maxwell equations in the 2-D case. Even though equation (1) cannot be derived from the Maxwell equations in 3-D for the case $\varepsilon_{r}(x) \neq$ const., still it was successfully used in $[8,27,31]$ to model the propagation of EM waves for experimental data, which are in 3-D.

Let $\Omega \subset \mathbb{R}^{3}$ be a convex bounded domain with the piecewise smooth boundary $\partial \Omega$. As it is always the case in CIPs, we need to assume a certain over-smoothness of the unknown coefficient. Let $d>1$ be a certain given number, which is the upper bound for our unknown coefficient $c$. Note that some a priori information about the unknown coefficient should be given in accordance with the Tikhonov concept for Ill-Posed Problems [40]. However, since we do not assume a smallness of the number $\widetilde{d}=d-1$, then a smallness assumption is not imposed on the function $c$. So, we assume that

$$
\begin{aligned}
& c(x) \in[1, d], c(x)=1 \text { for } x \in \mathbb{R}^{3} \backslash \Omega, \\
& c(x) \in C^{4}\left(\mathbb{R}^{3}\right) .
\end{aligned}
$$

The assumption that $c(x)$ is, in general, less outside of the domain of interest $\Omega$ than inside of it is because in our target application to imaging of antipersonnel plastic land mines the dielectric constant of mine-like targets is greater than the one in the background, see subsection 8.2. To simplify the presentation and also because of our target application, we now specify the domain $\Omega \subset \mathbb{R}^{3}$ as follows. Let $P>0$ be a constant. Below

$$
\begin{aligned}
\Omega & =\{(x, y, z):-P<x, y<P, z \in(0,2 P)\}, \partial \Omega=\cup_{i=1}^{3} \Gamma_{i}, \\
\Gamma_{1} & =\{(x, y, z):-P<x, y<P, z=0\}, \\
\Gamma_{2} & =\{(x, y, z): x, y= \pm P, z \in(0,2 P)\}, \Gamma_{3}=\bar{\Omega} \cap\{z=2 P\} .
\end{aligned}
$$

Inverse Problem. Suppose that the coefficient $c(x)$ in equation (1) satisfies conditions (3), (4) and is unknown in the domain $\Omega$. Determine $c(x)$ for $x \in \Omega$, assuming that the following functions $\bar{\varphi}_{0}(x, t)$ and $\bar{\varphi}_{1}(x, t)$ are known for a single source position $x_{0} \notin \bar{\Omega}$

$$
\left.u(x, t)\right|_{\Gamma_{1}}=\bar{\varphi}_{0}(x, t),\left.u_{z}(x, t)\right|_{\Gamma_{1}}=\bar{\varphi}_{1}(x, t), t \in(0, \infty) .
$$

In experiments usually only the function $\bar{\varphi}_{0}(x, t)$ is measured. One can approximately assume that this function is known at the entire plane $\{z=0\}$. Next, since by (3) and (5) the coefficient $c(x)=1$ for $z<0$, then solving the forward problem (1), (2) in the half space $\{z<0\}$ with the boundary condition $\left.u(x, t)\right|_{z=0}=\bar{\varphi}_{0}(x, t)$, one can uniquely determine the function $u(x, y, z, t)$ for $z<0, t>0$, which gives $\bar{\varphi}_{1}(x, t)$.

The next reasonable question to address is about the infinite rather than a finite time interval at which functions $\bar{\varphi}_{0}(x, t), \bar{\varphi}_{1}(x, t)$ are given in $(8)$. In our numerical procedure we use the Laplace transform with respect to $t$. Since the kernel $e^{-s t}, s>0$ of this transform decays rapidly when $t \rightarrow \infty$, then the integral over a finite time interval is approximately the same as the one over the infinite interval. This justifies our assumption $t \in(0, \infty)$ in (8). In addition, when working with experimental data, which were measured on a finite 
time interval $[8,27,31]$, we have observed that the assumption $t \in(0, \infty)$ does not affect the quality of solutions.

Remark 2.1. The question of the uniqueness of this CIP is a well known long standing problem. Currently it is addressed positively via the method of Carleman estimates only in the case when $\delta\left(x-x_{0}\right)$ in (2) is replaced with a function $f(x) \neq 0$ in $\bar{\Omega}[26,28]$. Still, because of applications, it makes sense to develop numerical methods for this CIP. Thus, we assume everywhere below that uniqueness of our Inverse Problem holds, also see Lemma 6.1.

\section{Laplace Transform}

Below $C^{k+\gamma}$ are Hölder spaces, where $k \geq 0$ is an integer and $\gamma \in(0,1)$. Let the function $c(x)$ be such that

$$
c \in C^{\gamma}\left(\mathbb{R}^{3}\right) \text { and conditions (3) hold. }
$$

Consider the Laplace transform $w$ of the function $u$. We are not concerned with inverting this transform, since we use it only to approximate the unknown coefficient $c(x)$. Thus,

$$
w(x, s)=\mathcal{L}_{1} u=\int_{0}^{\infty} u(x, t) e^{-s t} d t, \text { for } s \geq \underline{s}=\text { const. }>0
$$

where $\underline{s}>0$ is a certain number. In our numerical studies we choose $\underline{s}$ experimentally. We call the parameter $s$ pseudo frequency. The function $w$ is satisfies the following conditions

$$
\begin{aligned}
\Delta w-s^{2} c(x) w & =-\delta\left(x-x_{0}\right), x \in \mathbb{R}^{3}, \forall s \geq \underline{s}, \\
\lim _{|x| \rightarrow \infty} w(x, s) & =0, \forall s>\underline{s} .
\end{aligned}
$$

The condition (12) was established in subsection 2.2 of [6] for $\underline{s} \geq \underline{s}^{0}$, where the number

$\underline{s}^{0}=\underline{s}^{0}\left(\|c\|_{C^{\gamma}\left(\mathbb{R}^{3}\right)}\right)>0$ is sufficiently large. It was also proven in $[6]$ that for $s \geq \underline{s}^{0}$ the function $w(x, s)$ has the form

$$
\begin{aligned}
w & =w_{0}+\bar{w}, \text { where } \bar{w} \in C^{2+\gamma}\left(\mathbb{R}^{3}\right), \\
w_{0}(x, s) & =\frac{\exp \left(-s\left|x-x_{0}\right|\right)}{4 \pi\left|x-x_{0}\right|} .
\end{aligned}
$$

The function $w_{0}$ solves the problem $(11),(12)$ for the case $c(x) \equiv 1$. Theorem 6.17 of [21] about the smoothness of solutions of elliptic PDEs gives $\bar{w} \in C^{k+2+\gamma}\left(\mathbb{R}^{3}\right)$, if $c \in C^{k+\gamma}\left(\mathbb{R}^{3}\right)$.

In our algorithm as well as in the course of the proof of Theorem 7.1 we will construct solutions $w(x, \bar{s}):=w(x, \bar{s} ; c)$ of the problem (11), (12) with a certain $\bar{s}>1$ for different functions $c(x)$ satisfying $(9)$. These functions $c(x)$ will be calculated iteratively. We will not estimate $C^{\gamma}\left(\mathbb{R}^{3}\right)$ - norms of those functions $c(x)$. Hence, if constructing the sequence of functions $w(x, \bar{s} ; c)$ via solving the forward problem $(1),(2)$ and then calculating the 
integral (10) for $s:=\bar{s}$, then each of those functions $c(x)$ might require its own value $\underline{s}^{0}:=\underline{s}^{0}\left(\|c\|_{C^{\gamma\left(\mathbb{R}^{3}\right)}}\right)$, and there is no guarantee that $\underline{s}^{0}\left(\|c\|_{C^{\gamma}\left(\mathbb{R}^{3}\right)}\right)<\bar{s}$. However, since we will work only with the interval $s \in[\underline{s}, \bar{s}]$ for fixed values of parameters $\underline{s}, \bar{s}$, then we need to ensure that for each such function $c$ and for each value $s>0$ there exists unique solution of the form (13), (14) of the problem (11), (12). Thus, we now prove

Theorem 3.1. Let $x_{0} \notin \bar{\Omega}$, the function $c(x)$ satisfies condition (9) and also $c \in$ $C^{k+\gamma}\left(\mathbb{R}^{3}\right)$. Then for any $s>0$ there exists unique solution of the problem (11), (12) of the form (13), (14) with $\bar{w} \in C^{k+2+\gamma}\left(\mathbb{R}^{3}\right)$. Denote

$$
w^{(d)}(x, s)=\frac{\exp \left(-s \sqrt{d}\left|x-x_{0}\right|\right)}{4 \pi\left|x-x_{0}\right|} .
$$

the solution of the problem (11), (12) for $c(x) \equiv d$. Then

$$
w^{(d)}(x, s)<w(x, s) \leq w_{0}(x, s), \forall x \neq x_{0} .
$$

Proof. Consider the following parabolic Cauchy problem for $(x, t) \in \mathbb{R}^{3} \times(0, \infty)$

$$
c(x) v_{t}=\Delta v, v(x, 0)=\delta\left(x-x_{0}\right) .
$$

Let $v_{0}(x, t)$ be the solution of the problem (17) with $c \equiv 1$. Also, consider the function $\bar{v}(x, t)$,

$$
\begin{gathered}
v_{0}(x, t)=\frac{1}{(2 \sqrt{\pi t})^{3}} \exp \left(-\frac{\left|x-x_{0}\right|^{2}}{4 t}\right), \\
\bar{v}(x, t)=\int_{0}^{t}\left(v-v_{0}\right)(x, \tau) d \tau .
\end{gathered}
$$

Denote $b(x)=c(x)-1$. We obtain from (17) and (19)

$$
\Delta \bar{v}-c(x) \bar{v}_{t}=b(x) v_{0}, \bar{v}(x, 0)=0, \quad(x, t) \in \mathbb{R}^{3} \times(0, \infty) .
$$

Since $x_{0} \notin \bar{\Omega}$ and by (3) and $(9) b(x)=0$ outside of $\Omega$, then it follows from (18) that the right hand of $(20)$ does not have a singularity in $\mathbb{R}^{3} \times[0, \infty)$. Let $T, R>0$ be two arbitrary numbers and $B_{R}(T)=\{|x|<R\} \times(0, T)$. By (3), (9) and (18) $b(x) v_{0}(x, t) \geq 0$ for $(x, t) \in \mathbb{R}^{3} \times(0, \infty)$. Hence, applying to (20) the maximum principle of Theorem 1 of Chapter 2 of [20], we obtain $\max _{\bar{B}_{R}(T)} \bar{v}(x, t) \leq 0$. Since numbers $R, T>0$ are arbitrary, then

$$
\bar{v}(x, t) \leq 0 \text { in } \mathbb{R}^{3} \times[0, \infty) .
$$

On the other hand, Theorem 11 of Chapter 2 of [20] ensures that the fundamental solution of the parabolic equation is positive for $t>0$. Hence, (19) and (21) imply that

$$
\int_{0}^{t} v(x, \tau) d \tau \leq \int_{0}^{t} v_{0}(x, \tau) d \tau \text { and } v(x, t) \geq 0 .
$$


Next, consider the following version of the Laplace transform

$$
\mathcal{L}_{2} v=\int_{0}^{\infty} v(x, t) e^{-s^{2} t} d t
$$

By one of well known properties of the Laplace transform

$$
\mathcal{L}_{2}\left(\int_{0}^{t} f(\tau) d \tau\right)=\frac{1}{s^{2}} \mathcal{L}_{2} f
$$

for any appropriate function $f$. Formula (28) of section 4.5 of Tables [3] gives $\mathcal{L}_{2} v_{0}=w_{0}$. Fix a number $s>0$. Hence, (22)-(24) and Fubini theorem lead to

$$
\mathcal{L}_{2}\left(\int_{0}^{t} v(x, \tau) d \tau\right)=\frac{1}{s^{2}} \mathcal{L}_{2} v \leq \frac{1}{s^{2}} \mathcal{L}_{2}\left(v_{0}\right)=\frac{1}{s^{2}} w_{0}(x, s) .
$$

Hence, the integral (23) converges absolutely. Next, by (20) for any $A>0$

$$
\Delta \int_{0}^{A} \bar{v}(x, t) e^{-s^{2} t} d t=\int_{0}^{A} \Delta \bar{v}(x, t) e^{-s^{2} t} d t=\int_{0}^{A}\left[c \bar{v}_{t}+(c-1) v_{0}\right] e^{-s^{2} t} d t .
$$

Setting here $A \rightarrow \infty$ and using that by (19) $c \bar{v}_{t}+(c-1) v_{0}=c v-v_{0}$, we obtain

$$
\lim _{A \rightarrow \infty} \Delta \int_{0}^{A} \bar{v}(x, t) e^{-s^{2} t} d t=\lim _{A \rightarrow \infty} \int_{0}^{A} \Delta \bar{v}(x, t) e^{-s^{2} t} d t=c \mathcal{L}_{2} v-\mathcal{L}_{2} v_{0} .
$$

Hence, it follows from $(26)$ that $\Delta \mathcal{L}_{2}(\bar{v})$ and $\mathcal{L}_{2}(\Delta \bar{v})$ exist and $\Delta \mathcal{L}_{2}(\bar{v})=\mathcal{L}_{2}(\Delta \bar{v})$. Furthermore, by (19) and (24)-(26) $\Delta \mathcal{L}_{2}(\bar{v})=s^{-2} \Delta\left(\mathcal{L}_{2} v-\mathcal{L}_{2} v_{0}\right)=c \mathcal{L}_{2} v-\mathcal{L}_{2} v_{0}$. Hence, denoting $w:=\mathcal{L}_{2}(v)$ and using $\mathcal{L}_{2} v_{0}=w_{0}$ as well as $\Delta w_{0}-s^{2} w_{0}=-\delta\left(x-x_{0}\right)$, we obtain that the function $w$ satisfies equation (11).

We now prove (12) and also that

$$
w=w_{0}+\bar{w}, \text { where } \bar{w} \in C^{k+2+\gamma}\left(\mathbb{R}^{3}\right) .
$$

Since $c \bar{v}_{t}=\bar{v}_{t}+b \bar{v}_{t}$, then using (19) and (20), we obtain

$$
\bar{v}_{t}=\Delta \bar{v}-b(x) v, \bar{v}(x, 0)=0 .
$$

Since $b \in C^{k+\gamma}\left(\mathbb{R}^{3}\right)$ and $b(x)=0$ near $x_{0}$, then at least $v \in C^{2+\gamma, 1+\gamma / 2}\left(\mathbb{R}^{3} \times[0, T]\right), \forall T>0$ (formula (13.2) of Chapter 4 of [32]). Hence, by (27)

$$
\bar{v}(x, t)=-\int_{0}^{t} \int_{\Omega} v_{0}(x-\xi, t-\tau) b(\xi) v(\xi, \tau) d \xi d \tau .
$$


By (19), (23) and $(25) \mathcal{L}_{2} \bar{v}=s^{-2}\left(\mathcal{L}_{2} v-w_{0}\right)=s^{-2}\left(w-w_{0}\right)$. Hence, applying the Laplace transform $\mathcal{L}_{2}$ to both sides of (29) and using the convolution theorem, we obtain

$$
w(x, s)=w_{0}(x, s)-s^{2} \int_{\Omega} w_{0}(x-\xi) b(\xi) w(\xi, s) d \xi
$$

Iterating this integral equation once, we obtain

$$
\begin{aligned}
& w(x, s)=w_{0}(x, s)-s^{2} \int_{\Omega} w_{0}(x-\xi) w_{0}(\xi) b(\xi) d \xi \\
& +s^{4} \int_{\Omega} w_{0}(x-\xi) b(\xi) \int_{\Omega} w_{0}(\xi-\eta) b(\eta) w(\eta, s) d \eta .
\end{aligned}
$$

Since $b \in C^{\gamma}(\bar{\Omega})$, then the well known results for integral equations with kernels like $w_{0}(x-\xi)$ imply that

$$
\begin{gathered}
\left(\int_{\Omega} w_{0}(x-\xi) w_{0}(\xi) b(\xi) d \xi\right) \in C^{2+\gamma}\left(\mathbb{R}^{3}\right) \\
\left(\int_{\Omega} w_{0}(\xi-\eta) b(\eta) w(\eta, s) d \eta\right) \in C^{1}\left(\mathbb{R}^{3}\right) .
\end{gathered}
$$

By (14) and (30) functions $w(x, s), w_{0}(x, s), \bar{w}(x, s)=\left(w-w_{0}\right)(x, s)$ satisfy condition (12). Furthermore, it follows from (31)-(33) that (27) holds with $k=0$. Next, the above mentioned Theorem 6.17 of [21] ensures that (27) is true with $k \geq 0$. Thus, we have proven existence. Uniqueness is proven in subsection 2.2 of [6].

Finally we prove (16). Since $w=\mathcal{L}_{2}(v)$ and $v \geq 0, b \geq 0$, then the right inequality (16) follows from (30). Consider the function $\widetilde{w}^{(d)}(x, s)=w(x, s)-w^{(d)}(x, s)$. Then (11), (12) and (15) imply that

$$
\Delta \widetilde{w}^{(d)}-s^{2} c \widetilde{w}^{(d)}=s^{2}(c(x)-d) w^{(d)}, \lim _{|x| \rightarrow \infty} \widetilde{w}^{(d)}(x, s)=0 .
$$

By (13)-(15)

$$
\frac{\widetilde{w}^{(d)}(x, s)}{w^{(d)}(x, s)}=\exp \left[s(\sqrt{d}-1)\left|x-x_{0}\right|\right]\left(1-O\left(\left|x-x_{0}\right|\right)\right)>0, x \rightarrow x_{0}, x \neq x_{0} .
$$

Hence, there exists a sufficiently small number $\varepsilon>0$ such that

$$
\widetilde{w}^{(d)}(x, s)>0 \text { for } x \in\left\{\left|x-x_{0}\right| \leq \varepsilon\right\} .
$$


For $R>0$ consider the domain $B_{R, \varepsilon}=\left\{|x|<R,\left|x-x_{0}\right|>\varepsilon\right\}$. Assuming that $B_{R, \varepsilon} \neq \varnothing$, which is true for sufficiently large $R$, we obtain $\widetilde{w}^{(d)} \in C^{2+\gamma}\left(\bar{B}_{R, \varepsilon}\right)$ and $s^{2}(c(x)-d) w^{(d)} \leq 0$ in $B_{R, \varepsilon}$. Hence, applying the maximum principle to equation (34), we obtain $\min _{\bar{B}_{R, \varepsilon}} \widetilde{w}_{d} \geq$ $\min _{\partial B_{R, \varepsilon}} \widetilde{w}_{d}$. Setting $R \rightarrow \infty$ and using the second condition (34) as well as (35), we obtain $\min _{\left|x-x_{0}\right| \geq \varepsilon} \widetilde{w}^{(d)} \geq \min _{\left|x-x_{0}\right|=\varepsilon} \widetilde{w}_{d}>0$. Thus, $w(x, s)>w^{(d)}(x, s)$ for $x \neq x_{0}$.

To justify our new approximate mathematical model of subsection 6.3 , we need the asymptotic behavior of the function $w(x, s)$ at $s \rightarrow \infty$, see discussion in subsection 8.1 about the verification of conditions of Lemma 3.1 .

Lemma 3.1 [4]. Assume that conditions (3) and (4) hold. Let $u(x, t)$ be the solution of the problem (1), (2) and for sufficiently large values of $s>0$ let $w(x, s)=\mathcal{L}_{1} u$. Assume that geodesic lines, generated by the eikonal equation corresponding to the function $c(x)$ are regular, i.e. any two points in $\mathbb{R}^{3}$ can be connected by a single geodesic line. Let $l\left(x, x_{0}\right)$ be the length of the geodesic line connecting points $x$ and $x_{0}$. Then the following asymptotic behavior of the function $w$ and its derivatives takes place for $|\alpha| \leq 2, k=0,1, x \neq x_{0}$

$$
D_{x}^{\alpha} D_{s}^{k} w(x, s)=D_{x}^{\alpha} D_{s}^{k}\left\{\frac{\exp \left[-s l\left(x, x_{0}\right)\right]}{f\left(x, x_{0}\right)}\left[1+O\left(\frac{1}{s}\right)\right]\right\}, s \rightarrow \infty,
$$

where $f\left(x, x_{0}\right)$ is a certain function and $f\left(x, x_{0}\right) \neq 0$ for $x \neq x_{0}$.

Having the data at only one side $\Gamma_{1}$, as in (8), of the cube $\Omega$ is not sufficient for a good stability of the numerical solution. To provide a better stability, we now derive an approximate boundary condition for the function $\ln w$ at the rest $\Gamma_{2} \cup \Gamma_{3}$ of the boundary $\partial \Omega$. It follows from (14) and (30) that the function $w$ satisfies the radiation condition at infinity, $\lim _{|x| \rightarrow \infty}\left(\partial_{|x|} w+s w\right)(x)=0$, where $\partial_{|x|} w:=\partial_{r} w$ is understood in terms of sperical coordinates with the radius $r:=|x|$. Hence, assuming that the number $P$ in (5)-(7) is sufficiently large and keeping in mind that we work with an approximate mathematical model (subsections 6.2, 6.3), we impose the following approximate boundary condition at $\Gamma_{2} \cup \Gamma_{3}$

$$
\left.\left(\partial_{n} w+s w\right)\right|_{\Gamma_{2} \cup \Gamma_{3}}=0 .
$$

It follows from (37) that

$$
\left.\partial_{n}(\ln w(x, s))\right|_{\Gamma_{2} \cup \Gamma_{3}}=-s .
$$

Actually condition (38) is not an informative one. This is because it is independent on the target coefficient $c(x)$. Hence, it is logical to use two boundary conditions (8) as well as (38), since this should likely provide both a better reconstruction accuracy and a better stability.

Keeping in mind our target application of imaging of antipersonnel plastic land mines (section 1), we have verified the approximate boundary condition (37) computationally, both in 3-D and 2-D cases, as follows. For a variety of cases modeling our target application (subsection 8.2) we have computationally solved the forward problem for equation (11) in a domain $\widehat{\Omega}$, which was much larger than the domain $\Omega$ in $(5), \Omega \subset \widehat{\Omega}, \partial \Omega \cap \partial \widehat{\Omega}=\varnothing$. Because of (12), we have imposed the zero Dirichlet boundary condition at $\partial \widehat{\Omega}$. Next, we have solved equation (11) in the domain $\Omega$ with the boundary condition (37) at $\Gamma_{2} \cup \Gamma_{3}$. As to $\Gamma_{1}$, we have used the Dirichlet boundary condition, which was calculated from the above 
solution of the forward problem in $\widehat{\Omega}$. When doing so, we have used the same values of the parameter $s$ for which we have numerically solved our inverse problem. Comparison of these two solutions has consistently revealed that in a subdomain $\widetilde{\Omega} \subset \Omega$, whose boundary had a small distance from $\Gamma_{2} \cup \Gamma_{3}$, these two solutions have almost coincided. Thus, the above provides a numerical justification for the approximation (37), since mine-like inclusions of our interest are located in $\widetilde{\Omega}$, also see sub-subsection 8.1.1 for a relevant discussion.

\section{The Algorithm}

The algorithm of this section was described in detail in previous publications $[4,5]$ for the case of the complete data collection and in [30] for the case of backscattering data. However, since we need to refer to some parts of this algorithm in our convergence analysis in sections $5-7$, we need to outline it briefly here. We focus only on those items which we need in sections $5-7$.

\subsection{The sequence of elliptic equations}

We work only with the function $w(x, s), s>0$ assuming that conditions of Theorem 3.1 hold. Since by (15) $w>0$, then we can consider the function $v(x, s)=\ln w / s^{2}$. Let $q(x, s)=\partial_{s} v(x, s)$. An important point here is that

$$
v(x, s)=-\int_{s}^{\bar{s}} q(x, \tau) d \tau+V(x), V(x):=v(x, \bar{s}) .
$$

We call $V(x)$ the tail function. Here $\bar{s}>1$ is the truncation pseudo frequency which should be chosen in numerical experiments. Actually, $\bar{s}$ is one of regularization parameters of our method. Hence,

$$
V(x):=V(x, \bar{s})=\bar{s}^{-2} \ln w(x, \bar{s}) .
$$

Assuming that conditions of Lemma 3.1, we obtain

$$
\|V(x, \bar{s})\|_{C^{2+\gamma}(\bar{\Omega})}=O\left(\bar{s}^{-1}\right),\|q\|_{C^{2+\gamma}(\bar{\Omega})}=O\left(\bar{s}^{-2}\right), \bar{s} \rightarrow \infty .
$$

The function $q(x, s)$ satisfies a certain nonlinear integral differential PDE in which Volterralike integrals generated by (39) are involved. We have one equation with two unknown functions $q$ and $V$. The reason why we can approximate both of them is that we treat them differently: while we approximate the function $q$ via inner iterations "within" that equation, we approximate the function $V$ via outer iterations solving the problem (11), (12) and using (40) then. Boundary conditions (8), (38) are transformed in the following boundary conditions for $q(x, s)$

$$
\left.q\right|_{\Gamma_{1}}=\psi_{0}(x, s),\left.\partial_{z} q\right|_{\Gamma_{1}}=\psi_{1}(x, s),\left.\partial_{n} q\right|_{\Gamma_{2} \cup \Gamma_{3}}=s^{-2} .
$$


The problem of approximating functions $q$ and $V$ from that equation is the major difficulty of our method. We treat it via a layer stripping procedure with respect to $s$. Let $[\underline{s}, \bar{s}] \subset(0, \infty)$ be the $s$-interval where we approximate the function $q(x, s)$. In practical computations this interval should be chosen via numerical experiments conducted for a range of parameters, which is suitable for a specific application of ones interest. We approximate the function $q(x, s)$ as a piecewise constant function with respect to the pseudo frequency $s \in[\underline{s}, \bar{s}]$. That is, we assume that there exists a partition $\underline{s}=s_{N}<s_{N-1}<\ldots<s_{1}<s_{0}=\bar{s}$ of the interval $[\underline{s}, \bar{s}]$ with the sufficiently small grid step size $h=s_{i-1}-s_{i}$ such that $q(x, s)=q_{n}(x)$ for $s \in\left(s_{n}, s_{n-1}\right], n=1, \ldots, N$. Next, we multiply the above mentioned

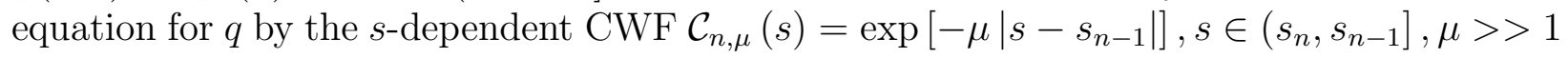
and integrate with respect to $s \in\left(s_{n}, s_{n-1}\right]$. The parameter $\mu$ should be chosen in numerical experiments. As a result, we obtain a coupled system of elliptic PDEs for functions $q_{n}$. Because of Volterra-like $s$-integrals in the original equation for $q$, this system can be solved sequentially starting from $q_{1}$. When solving these equations, we have $m \geq 1$ inner iterations for each $n$ with respect to the tail functions. Thus, for each $n \in[1, N]$ we obtain a finite sequence of functions $q_{n, k}, V_{n, k}, k \in[1, m]$. Those equations and boundary conditions for functions $q_{n, k}$ are

$$
\begin{gathered}
\Delta q_{n, k}-A_{1, n}\left(h \sum_{j=0}^{n-1} \nabla q_{j}-\nabla V_{n, k}\right) \nabla q_{n, k}=-A_{2, n} h^{2}\left(\sum_{j=0}^{n-1} \nabla q_{j}\right)^{2} \\
+2 A_{2, n} \nabla V_{n, k}\left(h \sum_{j=0}^{n-1} \nabla q_{j}\right)-A_{2, n}\left(\nabla V_{n, k}\right)^{2}, x \in \Omega,(n, k) \in[1, N] \times[1, m], \\
\left.q_{n, k}\right|_{\Gamma_{1}}=\psi_{0, n}(x),\left.\partial_{z} q_{n, k}\right|_{\Gamma_{1}}=\psi_{1, n}(x),\left.\partial_{n} q_{n, k}\right|_{\Gamma_{2} \cup \Gamma_{3}}=\left(s_{n} s_{n-1}\right)^{-1} .
\end{gathered}
$$

Functions $\psi_{0, n}(x)$ and $\psi_{1, n}(x)$ are averages over the interval $\left(s_{n}, s_{n-1}\right)$ of respectively functions $\psi_{0}(x, s)$ and $\psi_{1}(x, s)$ in $(42)$ and are therefore approximate boundary conditions. The third boundary condition in (44) is the average of the function $s^{-2}$ in (42) over the interval $\left(s_{n}, s_{n-1}\right)$. In $(43) A_{i, n}=A_{i, n}(h, \mu)>0, i=1,2$ are known parameters, see specific formulas in [4]. It is convenient to set in (43)

$$
q_{0} \equiv 0
$$

\section{Remarks 4.1:}

1. In fact, one should also have the nonlinear term $B_{n}(\mu, h)\left(\nabla q_{n, k}\right)^{2}$ in the right hand side of equation (43) with a certain parameter $B_{n}(\mu, h)$ [4]. However, because of the presence of the CWF we have $\left|B_{n}(\mu, h)\right| \leq 8 \bar{s}^{2} / \mu$ for $\mu h \geq 1$ [4]. We have used $\mu=50$ in our computations. Hence, assuming that $\mu>>1$, we ignore this term. This allows us to solve a linear problem for each $q_{n, k}$. We have also conducted numerical experiments for the case when this term is not ignored. In this case we have also performed iterations with respect to this term. However, results of those numerical studies have shown that the influence of this term is negligible for $\mu \geq 50$. 
2. In principle one can avoid using the CWF via choosing the size $h$ of each subinterval $\left(s_{n}, s_{n-1}\right)$ to be very small. This would again result in a small multiplier at $\left(\nabla q_{n}\right)^{2}$. However the number $N$ of subintervals would increase in this case, which would make it necessary to solve more problems for functions $q_{n}$. Hence, both the computational time and the computational error would increase.

3. Setting $B_{n}\left(\nabla q_{n, k}\right)^{2}:=0$ for large values of $\mu$ is not a linearization. The nonlinearity actually surfaces in the iterative process in those terms of (43) which contain $q_{j}$ and $V_{n, k}$. Indeed, these terms contain products $\nabla q_{j} \nabla q_{n, k}$. Also, tails $V_{n, k}$ depend nonlinearly on functions $q_{j}, q_{n, k}$, see (50) and (51).

4. In our analytical derivation we use the third condition (42) at $\Gamma_{3}$ only at $s=\bar{s}$, where $\bar{s}$ is the highest value of $s$ we use in our algorithm, see (104), (105) and Lemma 7.1. However, we use the third condition (42) at $\Gamma_{2}$ for all values of $s$ in our convergence analysis. As to the numerical studies, our computational experience has shown that it is better for the stability to use the third condition (42) at $\Gamma_{2} \cup \Gamma_{3}$ for all values of $s$, and this is what we do in our numerical testing, see subsection 8.1 for a relevant discussion.

5. In addition, if using the Laplace transform of only one of conditions (8) combined with the third condition (42), then there is an analytical difficulty here, compared with $[4,5]$. Indeed, in the convergence analysis of $[4,5]$ the boundary $\partial \Omega \in C^{3}$, which enabled one to use Schauder theorem. However, the boundary of our cube $\Omega$ in (5)-(7) is not smooth. Hence, the existence of a smooth solution for the corresponding elliptic boundary value problem for the function $q_{n, k}$ in section 4 cannot be guaranteed in this case.

6. In summary, it follows from the above two remarks that it is optimal in computations to use the Laplace transforms of both boundary conditions (8) as well as the third condition (42). The over-determination in boundary conditions (8) logically leads to the QRM.

The iterative process of the next subsection reconstructs iterative approximations $c_{n, k}(x) \in$ $C^{\gamma}(\bar{\Omega})$ of the function $c(x)$ and $c_{n, k}(x) \in[1, d]$ in $\bar{\Omega}$. On the other hand, to iterate with respect to the tails, we need to solve the forward problem (11), (12) on each iterative step. To do this, we extend each function $c_{n, k}(x)$ outside of the domain $\Omega$. So that the resulting function $\widehat{c}_{n, k}(x)=1$ outside of $\Omega, \widehat{c}_{n, k}(x)=c_{n, k}(x)$ in a subdomain $\Omega^{\prime} \subset \Omega, \widehat{c}_{n, k}(x) \in[1, d], \forall x \in \mathbb{R}^{3}$ and $\widehat{c}_{n, k} \in C^{\gamma}\left(\mathbb{R}^{3}\right)$. When estimating solutions resulting from the QRM, we need to use a Carleman estimate, which, however, estimates the solution only in a subdomain of the original domain. The way out of this is that in applications one can always choose such a domain of interest which is a little bit bigger than the one where the coefficient is unknown, and we need this in our convergence analysis in section 7 . These considerations prompt us to apply the construction described below in this subsection.

Let $P$ be the number in (5)-(7) and $P_{0}=$ const. $\in(0, P)$. Denote $\Omega_{P_{0}}=\Omega \cap\left\{z \in\left(0, P_{0}\right)\right\}$. We will assume below that $c(x)=1, \forall x \in \mathbb{R}^{3} \backslash \Omega_{P_{0}}$. Consider a subdomain $\Omega^{\prime} \subset \Omega_{P_{0}}$. Choose a function $\chi_{1}(x) \in C^{\infty}\left(\mathbb{R}^{3}\right)$ such that

$$
\chi_{1}(x)=\left\{\begin{array}{c}
1 \text { in } \Omega^{\prime}, \\
\text { between } 0 \text { and } 1 \text { in } \Omega_{P_{0}} \backslash \Omega^{\prime}, \\
0 \text { outside of } \Omega_{P_{0}} .
\end{array}\right.
$$


The existence of such functions $\chi_{1}(x)$ is well known from the Real Analysis course. Define the target extension of the function $c_{n, k}$ as

$\widehat{c}_{n, k}(x):=\left(1-\chi_{1}(x)\right)+\chi_{1}(x) c_{n, k}(x), \forall x \in \mathbb{R}^{3}$. Hence, $\widehat{c}_{n, k}(x)=1$ outside of $\Omega_{P_{0}}$ and $\widehat{c}_{n, k} \in C^{\gamma}\left(\mathbb{R}^{3}\right)$. Using $c_{n, k}(x) \in[1, d], \forall x \in \bar{\Omega}$, it is easy to verify that $\widehat{c}_{n, k}(x) \in[1, d], \forall x \in$ $\mathbb{R}^{3}$.

\subsection{The iterative process}

First, we choose an initial tail function $V_{1,1}(x) \in C^{2+\gamma}(\bar{\Omega})$. Our choice of this function is described in subsection 6.3 and it is different from ones used in previous publications on this method. Let $m \geq 1$ be an integer which we choose in numerical experiments. For each $n \in[1, N]$ we have $m$ inner iterations with respect to the tails via computing functions $q_{n, k}, V_{n, k}, k \in[1, m]$.

Step $n^{k}$, where $n \in[1, N], k \in[1, m]$. Recall that by (45) $q_{0} \equiv 0$. Suppose that functions $q_{j} \in H^{5}(\Omega), j=1, \ldots, n-1$ and tails $V_{1}, \ldots, V_{n-1}, V_{n, k} \in C^{2+\gamma}(\bar{\Omega})$ are constructed. First, we change the operator and the right hand side of (43) as described in subsection 4.3. To construct the function $q_{n . k}$, we use the QRM (subsection 4.3) to find the "least squares" solution of the perturbed boundary value problem (43), (44). Hence, we obtain the function $q_{n, k} \in H^{5}(\Omega)$. To reconstruct an approximation $c_{n, k}(x)$ for the function $c(x)$, we first use our discrete analog of (39) to calculate an approximation for the function $v\left(x, s_{n}\right)$ and then calculate an approximation $\bar{c}_{n, k}$ for the function $c$. Specifically,

$$
\begin{aligned}
v_{n, k}\left(x, s_{n}\right) & =-h q_{n, k}(x)-h \sum_{j=0}^{n-1} q_{j}(x)+V_{n, k}(x), x \in \Omega_{P_{0}}, \\
\bar{c}_{n, k}(x) & =\Delta v_{n, k}\left(x, s_{n}\right)+s_{n}^{2}\left|\nabla v_{n, k}\left(x, s_{n}\right)\right|^{2}, x \in \Omega_{P_{0}} .
\end{aligned}
$$

Since we assume in (3) that the exact solution of our Inverse Problem $c^{*} \in[1, d]$ and since the computed function $\bar{c}_{n, k}$ in (47) does not necessarily satisfy this requirement, we set

$$
c_{n, k}(x)=\left\{\begin{array}{c}
\bar{c}_{n, k}(x), \text { if } \bar{c}_{n, k}(x) \in[1, d], x \in \Omega_{P_{0}} \\
1, \text { if } \bar{c}_{n, k}(x)<1, x \in \Omega_{P_{0}} \\
d, \text { if } \bar{c}_{n, k}(x)>d, x \in \Omega_{P_{0}}
\end{array}\right.
$$

Since functions $q_{j}, q_{n, k} \in H^{5}(\Omega)$, then the embedding theorem implies that $q_{j}, q_{n, k} \in C^{3}(\bar{\Omega})$. In addition, the tail function $V_{n, k} \in C^{2+\gamma}(\bar{\Omega})$. Hence, (46)-(48) imply that

$$
c_{n, k} \in C^{\gamma}(\bar{\Omega}) .
$$

Next, we construct the function $\widehat{c}_{n, k}(x)$ as explained in subsection 4.1. By (49) the function $\widehat{c}_{n, k} \in C^{\gamma}\left(\mathbb{R}^{3}\right)$. Next, we solve the forward problem (11), (12) with $c(x):=\widehat{c}_{n, k}(x)$ for $s:=\bar{s}$ and obtain the function $w_{n, k}(x, \bar{s})$. Existence and uniqueness of the function $w_{n, k}(x, \bar{s})$ in the form (13), (14) are guaranteed by Theorem 3.1. We set for the new tail

$$
V_{n, k+1}(x)=\bar{s}^{-2} \ln w_{n, k}(x, \bar{s}) \in C^{2+\gamma}(\bar{\Omega}) \text { if } k<m .
$$


And if $k=m$, then we set $c_{n}(x):=c_{n, m}(x), q_{n}(x):=q_{n, m}(x)$. And for the tail

$$
V_{n}(x):=V_{n, m+1}(x):=\bar{s}^{-2} \ln w_{n, m}(x, \bar{s}):=V_{n+1,1}(x) \text { for } x \in \Omega .
$$

\subsection{The quasi-reversibility method}

Since the Carleman estimate of section 5 estimates the target function only in a subdomain of the domain $\Omega$, we introduce the function $\chi_{2}(x)$ which is the characteristic function of the subdomain $\Omega_{P_{0}}, \chi_{2}(x)=1$ in $\Omega_{P_{0}}$ and $\chi_{2}(x)=0$ in $\mathbb{R}^{3} \backslash \Omega_{P_{0}}$. Denote

$$
\begin{aligned}
a_{n, k}(x)= & A_{1, n}\left(\chi_{2}(x) h \sum_{j=0}^{n-1} \nabla q_{j}-\nabla V_{n, k}\right) \\
H_{n, k}(x)= & -A_{2, n} h^{2}\left(\sum_{j=0}^{n-1} \nabla q_{j}\right)^{2} \chi_{2}(x)+2 A_{2, n} \nabla V_{n, k}\left(h \sum_{j=0}^{n-1} \nabla q_{j}\right) \chi_{2}(x) \\
& -A_{2, n}\left(\nabla V_{n, k}\right)^{2} .
\end{aligned}
$$

Hence, the function $H_{n, k}(x)$ is the perturbed right hand side of equation (43). Note that $H_{n, k} \in L_{2}(\Omega)$. The perturbed boundary value problem (43), (44) is

$$
\begin{gathered}
\Delta q_{n, k}-a_{n, k} \nabla q_{n, k}=H_{n, k} \\
\left.q_{n, k}\right|_{\Gamma_{1}}=\psi_{0, n}(x),\left.\partial_{z} q_{n, k}\right|_{\Gamma_{1}}=\psi_{1, n}(x),\left.\partial_{n} q_{n, k}\right|_{\Gamma_{2} \cup \Gamma_{3}}=\left(s_{n} s_{n-1}\right)^{-1} .
\end{gathered}
$$

Since we have two boundary conditions rather then one at $\Gamma_{1}$, we find the "least squares" solution of the problem (54), (55) via the QRM. Specifically, we minimize the following Tikhonov functional

$$
J_{n, k}^{\alpha}(u)=\left\|\Delta u-a_{n, k} \nabla u-H_{n, k}\right\|_{L_{2}(\Omega)}^{2}+\alpha\|u\|_{H^{5}(\Omega)}^{2},
$$

subject to the boundary conditions (55). Here $\alpha \in(0,1)$ is the small regularization parameter. Let $\bar{u}(x)$ be the unique minimizer of this functional, which is guaranteed by Lemma 5.2. Then we set $q_{n, k}(x):=\bar{u}(x)$. Local minima do not occur here since (56) is the sum of square norms of two expressions, both of which are linear with respect to $u$. The second term in the right hand side of (56) is the Tikhonov regularization term. We use the $H^{5}(\Omega)$-norm here in order to ensure that the minimizer $u:=q_{n, k} \in C^{3}(\bar{\Omega})$. It was shown above that the latter implies (49). We call the minimizer $\bar{u}(x)$ of the functional $J_{n, k}^{\alpha}(u)$ the QRM solution of the problem (54), (55).

\section{Estimates for the QRM}

For brevity we scale variables in such a way that in (5)-(7) $P=1 / 2$ in sections $5-7$

$$
\Omega=\{x=(x, y, z):(x, y) \in(-1 / 4,1 / 4) \times(-1 / 4,1 / 4), z \in(0,1 / 2)\},
$$


Let $\lambda, \nu>2$ be two parameters. Introduce the $z$-dependent CWF $K(z)$,

$$
K(z):=K_{\lambda, \nu}(z)=\exp \left(\lambda \rho^{-\nu}\right), \text { where } \rho(z)=z+1 / 4, z>0 .
$$

This CWF is different from the ones previously used for Carleman estimates for elliptic PDEs $[16,28,34]$. Note that $\rho(z) \in(0,3 / 4)$ in $\Omega$ and $\left.\rho(z)\right|_{\Gamma_{3}}=3 / 4$. Let the number $\varkappa \in(1 / 3,1)$. Denote $\Omega_{\varkappa}=\{x \in \Omega: \rho(z)<3 \varkappa / 4\}$. Hence, if $\varkappa_{1}<\varkappa_{2}$, then $\Omega_{\varkappa_{1}} \subset \Omega_{\varkappa_{2}}$. Also, $\Omega_{1}=\Omega$ and $\Omega_{1 / 3}=\varnothing$. In addition, $K^{2}(z) \geq \exp \left[2 \lambda((4 / 3) \varkappa)^{\nu_{0}}\right]$ in $\Omega_{\varkappa}$. Everywhere below $C>0$ denotes different generic constants which are independent on the domain $\Omega$ in $(57),(\cdot, \cdot)$ denotes the scalar product in $L_{2}(\Omega)$ and $[\cdot, \cdot]$ denotes the scalar product in $H^{5}(\Omega)$.

Lemma 5.1. Fix a number $\nu:=\nu_{0}>2$. Consider functions $u \in H^{3}(\Omega)$ such that (see $(5)-(7))$

$$
\left.u\right|_{\Gamma_{1}}=\left.u_{z}\right|_{\Gamma_{1}}=\left.\partial_{n} u\right|_{\Gamma_{2}}=0 .
$$

Then there exists a constant $C>0$ such that for all $\lambda>2$ the following Carleman estimate is valid for all these functions

$$
\begin{aligned}
\int_{\Omega}(\Delta u)^{2} K^{2} d x \geq & \frac{C}{\lambda} \sum_{|\alpha|=2} \int_{\Omega}\left(D^{\alpha} u\right)^{2} K^{2} d x+C \int_{\Omega}\left[\lambda(\nabla u)^{2}+\lambda^{3} u^{2}\right] K^{2} d x \\
& -C \lambda^{3}\|u\|_{H^{3}(\Omega)}^{2} \exp \left[2 \lambda(4 / 3)^{\nu_{0}}\right] .
\end{aligned}
$$

Proof. It is convenient to initially assume that $\nu>2$ is an arbitrary number. We have

$$
\begin{aligned}
(\Delta u)^{2} K^{2}= & \left(u_{x x}^{2}+u_{y y}^{2}+u_{z z}^{2}+2 u_{x x} u_{z z}+2 u_{x x} u_{y y}+2 u_{y y} u_{z z}\right) K^{2} \\
= & \left(u_{x x}^{2}+u_{y y}^{2}+u_{z z}^{2}\right) K^{2} \\
& +\partial_{x}\left(2 u_{x} u_{z z} K^{2}+2 u_{x} u_{y y} K^{2}\right)+\partial_{y}\left(2 u_{y} u_{z z} K^{2}\right) \\
& -2 u_{x} u_{z z x} K^{2}-2 u_{x} u_{y y x} K^{2}-2 u_{y} u_{z z y} K^{2} \\
= & \left(u_{x x}^{2}+u_{y y}^{2}+u_{z z}^{2}\right) K^{2}+\partial_{x}\left(2 u_{x} u_{z z} K^{2}+2 u_{x} u_{y y} K^{2}\right)+\partial_{y}\left(2 u_{y} u_{z z} K^{2}\right) \\
& +\partial_{y}\left(-2 u_{x} u_{x y} K^{2}\right)+2 u_{x y}^{2} K^{2} \\
& +\partial_{z}\left(-2 u_{x} u_{x z} K^{2}\right)+2 u_{x z}^{2} K^{2}-4 \lambda \nu \rho^{-\nu-1} u_{x} u_{x z} K^{2} \\
& +\partial_{z}\left(-2 u_{y} u_{y z} K^{2}\right)+2 u_{y z}^{2} K^{2}-4 \lambda \nu \rho^{-\nu-1} u_{y} u_{y z} K^{2}
\end{aligned}
$$

Thus, we have obtained that

$$
\begin{aligned}
(\Delta u)^{2} K^{2}= & \left(u_{x x}^{2}+u_{y y}^{2}+u_{z z}^{2}+2 u_{x y}^{2}+2 u_{x z}^{2}+2 u_{y z}^{2}\right) K^{2} \\
& -4 \lambda \nu \rho^{-\nu-1}\left(u_{x} u_{x z}+u_{y} u_{y z}\right) K^{2}+\partial_{x}\left[2\left(u_{x} u_{z z}+u_{x} u_{y y}\right) K^{2}\right] \\
& +\partial_{y}\left[2\left(u_{y} u_{z z}-u_{x} u_{x y}\right) K^{2}\right]+\partial_{z}\left[-2\left(u_{x} u_{x z}+u_{y} u_{y z}\right) K^{2}\right]
\end{aligned}
$$

Using the Cauchy-Schwarz inequality " $\forall \varepsilon>0$ ", $2 a b \geq-\varepsilon a^{2}-b^{2} / \varepsilon$ and taking $\varepsilon=1 / 4$, we obtain

$$
-4 \lambda \nu \rho^{-\nu-1}\left(u_{x} u_{x z}+u_{y} u_{y z}\right) K^{2} \geq-\left(u_{x z}^{2}+u_{y z}^{2}\right) K^{2}-4 \lambda^{2} \nu^{2} \rho^{-2 \nu-2}(\nabla u)^{2} K^{2} .
$$


Hence, (59) implies that

$$
\begin{aligned}
(\Delta u)^{2} K^{2} \geq & \sum_{|\alpha|=2}\left(D^{\alpha} u\right)^{2} K^{2}-4 \lambda^{2} \nu^{2} \rho^{-2 \nu-2}(\nabla u)^{2} K^{2}+\partial_{x}\left[2 u_{x}\left(u_{z z}+u_{y y}\right) K^{2}\right] \\
& +\partial_{y}\left[2\left(u_{y} u_{z z}-u_{x} u_{x y}\right) K^{2}\right]+\partial_{z}\left[-2\left(u_{x} u_{x z}+u_{y} u_{y z}\right) K^{2}\right] .
\end{aligned}
$$

Consider a new function $v=u K$. Substituting $u=v K^{-1}$, we obtain

$$
\begin{array}{r}
(\Delta u)^{2} \rho^{\nu+1} K^{2}=\left(y_{1}+y_{2}+y_{3}\right)^{2} \rho^{\nu+1} \geq 2 y_{2}\left(y_{1}+y_{3}\right) \rho^{\nu+1}, \\
y_{1}=\Delta v, y_{2}=2 \lambda \nu \rho^{-\nu-1} v_{z}, y_{3}=(\lambda \nu)^{2} \rho^{-2 \nu-2}\left(1-(\nu+1)(\lambda \nu)^{-1} \rho^{\nu}\right) v .
\end{array}
$$

We have

$$
2 y_{1} y_{2} \rho^{\nu+1}=\partial_{x}\left(4 \lambda \nu v_{z} v_{x}\right)+\partial_{y}\left(4 \lambda \nu v_{z} v_{y}\right)+\partial_{z}\left[2 \lambda \nu\left(v_{z}^{2}-v_{x}^{2}-v_{y}^{2}\right)\right] .
$$

Next, by (61) and (62)

$$
\begin{aligned}
2 y_{2} y_{3} \rho^{\nu+1}= & 4(\lambda \nu)^{3}\left(\rho^{-2 \nu-2}-(\nu+1)(\lambda \nu)^{-1} \rho^{-\nu-2}\right) v_{z} v \\
= & \partial_{z}\left[2(\lambda \nu)^{3}\left(\rho^{-2 \nu-2}-(\nu+1)(\lambda \nu)^{-1} \rho^{-\nu-2}\right) v^{2}\right] \\
& +4(\lambda \nu)^{3}(\nu+1) \rho^{-2 \nu-3}\left(1-(\nu+2)(2 \lambda \nu)^{-1} \rho^{\nu}\right) v^{2} \\
\geq & 2 \lambda^{3} \nu^{4} \rho^{-2 \nu-3} v^{2}+\partial_{z}\left[2(\lambda \nu)^{3}\left(\rho^{-2 \nu-2}-(\nu+1)(\lambda \nu)^{-1} \rho^{-\nu-2}\right) v^{2}\right] .
\end{aligned}
$$

Summing up (63) with (64), using (61) and the backwards substitution $u=v K$, we obtain

$$
(\Delta u)^{2} \rho^{\nu+1} K^{2} \geq 2 \lambda^{3} \nu^{4} \rho^{-2 \nu-3} u^{2} K^{2}+\partial_{x} U_{1}+\partial_{y} U_{2}+\partial_{z} U_{3},
$$

where the following estimates are valid for functions $U_{1}, U_{2}, U_{3}$

$$
\begin{aligned}
& \left|U_{1}\right| \leq C \lambda \nu\left|u_{x}\right|\left(\left|u_{z}\right|+\lambda \nu \rho^{-\nu-1}|u|\right) K^{2} \\
& \left|U_{2}\right| \leq C \lambda \nu\left|u_{y}\right|\left(\left|u_{z}\right|+\lambda \nu \rho^{-\nu-1}|u|\right) K^{2} \\
& \left|U_{3}\right| \leq C \lambda \nu\left(|\nabla u|^{2}+\lambda^{2} \nu^{2} \rho^{-2 \nu-2} u^{2}\right) K^{2}
\end{aligned}
$$

We now need to incorporate the term $\lambda(\nabla u)^{2} K^{2}$ in the right hand side of the Carleman estimate. Hence, we continue as follows:

$$
\begin{aligned}
-\lambda \nu u \Delta u K^{2}= & \partial_{x}\left(-\lambda \nu u u_{x} K^{2}\right)+\partial_{y}\left(-\lambda \nu u u_{y} K^{2}\right)+\partial_{z}\left(-\lambda \nu u u_{z} K^{2}\right) \\
& +\lambda \nu(\nabla u)^{2} K^{2}-2 \lambda^{2} \nu^{2} \rho^{-\nu-1} u_{z} u K^{2}=\lambda \nu(\nabla u)^{2} K^{2} \\
& -2 \lambda^{3} \nu^{3} \rho^{-2 \nu-2}\left(1+(\nu+1)(2 \lambda \nu)^{-1} \rho^{\nu}\right) u^{2} K^{2}+\partial_{x} U_{4}+\partial_{y} U_{5}+\partial_{z} U_{6},
\end{aligned}
$$

Hence,

$$
-\lambda \nu u \Delta u K^{2} \geq \lambda \nu(\nabla u)^{2} K^{2}-4 \lambda^{3} \nu^{3} \rho^{-2 \nu-2} u^{2} K^{2}+\partial_{x} U_{4}+\partial_{y} U_{5}+\partial_{z} U_{6},
$$




$$
U_{4}=-\lambda \nu u u_{x} K^{2}, U_{5}=-\lambda \nu u u_{y} K^{2},\left|U_{6}\right| \leq C\left(\lambda \nu u_{z}^{2}+\lambda^{2} \nu^{2} \rho^{-\nu-1} u^{2}\right) K^{2} .
$$

Sum up (65) and (67), take into account (66) and (68) as well as the fact that

$$
2 \lambda^{3} \nu^{4} \rho^{-2 \nu-3}-4 \lambda^{3} \nu^{3} \rho^{-2 \nu-2}=2 \lambda^{3} \nu^{4} \rho^{-2 \nu-3}\left(1-\rho(2 \nu)^{-1}\right)>\lambda^{3} \nu^{4} \rho^{-2 \nu-3} .
$$

We obtain

$$
\begin{array}{r}
(\Delta u)^{2} K^{2}-\lambda \nu u \Delta u K^{2} \geq \\
\lambda \nu(\nabla u)^{2} K^{2}+\lambda^{3} \nu^{4} \rho^{-2 \nu-3} u^{2} K^{2}+\partial_{x} U_{7}+\partial_{y} U_{8}+\partial_{z} U_{9}, \\
\left|U_{7}\right| \leq C \lambda \nu\left|u_{x}\right|\left(\left|u_{z}\right|+\lambda \nu \rho^{-\nu-1}|u|\right) K^{2} \\
\left|U_{8}\right| \leq C \lambda \nu\left|u_{y}\right|\left(\left|u_{z}\right|+\lambda \nu \rho^{-\nu-1}|u|\right) K^{2} \\
\left|U_{9}\right| \leq C \lambda \nu\left(|\nabla u|^{2}+\lambda^{2} \nu^{2} \rho^{-2 \nu-2} u^{2}\right) K^{2} .
\end{array}
$$

Fix the number $\nu:=\nu_{0}>2$. Then we can incorporate $\nu_{0}$ in $C$ and can regard that $\rho^{\nu_{0}+1}<$ $C$, since $\rho^{\nu_{0}+1}<1$. By the Cauchy-Schwarz inequality $-\lambda \nu u \Delta u K^{2} \leq(\Delta u)^{2} \rho^{\nu+1} K^{2} / 2+$ $\lambda^{2} \nu^{2} \rho^{-\nu-1} u^{2} K^{2} / 2$. Hence, we obtain from (69)-(72)

$$
(\Delta u)^{2} K^{2} \geq C\left[\lambda(\nabla u)^{2}+\lambda^{3} u^{2}\right] K^{2}+\partial_{x} U_{7}+\partial_{y} U_{8}+\partial_{z} U_{9}
$$

We now set in (60) $\nu:=\nu_{0}$, divide it by $\lambda p$ with a positive constant $p=p\left(\nu_{0}\right)$ such that $4 \nu_{0}^{2} \rho^{-2 \nu_{0}-2} / m \leq C / 2$, add the resulting inequality to (73) and take into account (70)-(72). Then with a new constant $C$ we obtain the following pointwise Carleman estimate for the Laplace operator in the domain $\Omega$

$$
\begin{aligned}
(\Delta u)^{2} K^{2} \geq & \frac{C}{\lambda} \sum_{|\alpha|=2}\left(D^{\alpha} u\right)^{2} K^{2}+C\left[\lambda(\nabla u)^{2}+\lambda^{3} u^{2}\right] K^{2} \\
& +\partial_{x} U_{10}+\partial_{y} U_{11}+\partial_{z} U_{12}, \\
\left|U_{10}\right| \leq & C \lambda\left|u_{x}\right|\left(\left|u_{z z}\right|+\left|u_{y y}\right|+\left|u_{z}\right|+\lambda|u|\right) K^{2}, \\
\left|U_{11}\right| \leq & C\left[\lambda\left|u_{y}\right|\left(\left|u_{z z}\right|+\left|u_{z}\right|+\lambda|u|\right)+\left|u_{x y}\right|\left|u_{x}\right|\right] K^{2} \\
\left|U_{12}\right| \leq & C \lambda\left[\left|u_{x z}\right|^{2}+\left|u_{y z}\right|^{2}+|\nabla u|^{2}+\lambda^{2} u^{2}\right] K^{2} .
\end{aligned}
$$

We now integrate the formula (74) over the rectangle $\Omega$ using the Gauss formula. It is important that because of (58) and estimates (75)-(77), resulting boundary integrals over $\Gamma_{1}$ and $\Gamma_{2}$ will be zero. We obtain

$$
\begin{aligned}
\int_{\Omega}(\Delta u)^{2} K^{2} d x \geq & \frac{C}{\lambda} \sum_{|\alpha|=2} \int_{\Omega}\left(D^{\alpha} u\right)^{2} K^{2} d x+C \int_{\Omega}\left[\lambda(\nabla u)^{2}+\lambda^{3} u^{2}\right] K^{2} d x \\
& -C \lambda \int_{\Gamma_{3}}\left[\left|u_{x z}\right|^{2}+\left|u_{y z}\right|^{2}+|\nabla u|^{2}+\lambda^{2} u^{2}\right] K^{2} d S .
\end{aligned}
$$

Note that $K^{2}(1 / 2)=\left.K^{2}(z)\right|_{\Gamma_{3}}=\min _{\bar{\Omega}} K^{2}(z)=\exp \left[2 \lambda(4 / 3)^{\nu_{0}}\right]$. Thus,

$$
\int_{\Gamma_{3}} \lambda\left[\left|u_{x z}\right|^{2}+\left|u_{y z}\right|^{2}+|\nabla u|^{2}+\lambda^{2} u^{2}\right] K^{2} d x \leq C \lambda^{3}\|u\|_{H^{3}(\Omega)}^{2} \exp \left[2 \lambda(4 / 3)^{\nu_{0}}\right] .
$$


Substituting this in (78), we obtain the estimate of this lemma.

A peculiarity of the proof of Lemma 5.1 is that when integrating the pointwise Carleman estimate (74) over the domain $\Omega$, we should take into account only one (rather than conventional two) zero boundary condition (58) at $\Gamma_{2}$. This requires a careful evaluation of terms under signs of derivatives $\partial_{x}, \partial_{y}$ which was not done before.

We now establish both existence and uniqueness of the minimizer of the functional (56). Denote $a_{n, k}^{(i)}(x), i=1,2,3$ components of the vector function $a_{n, k}(x)$ in (52), (54). Let

$$
\left\|a_{n, k}^{(i)}\right\|_{L_{\infty}(\Omega)} \leq M, M=\text { const. }>0, i=1,2,3 .
$$

Lemma 5.2. Assume that there exists a function $\Phi \in H^{5}(\Omega)$ satisfying boundary conditions (55), except of maybe at $\Gamma_{3}$, and condition (79) holds. Then there exists unique minimizer $u \in H^{5}(\Omega)$ of the functional (56). Furthermore, with a constant $C_{1}=C_{1}(M)>0$

$$
\|u\|_{H^{5}(\Omega)} \leq C_{1} \alpha^{-1 / 2}\left(\left\|H_{n, k}\right\|_{L_{2}(\Omega)}+\|\Phi\|_{H^{5}(\Omega)}\right) .
$$

Proof. Let $u$ be a minimizer of $J_{n, k}^{\alpha}(u)$ satisfying boundary conditions (55). Let $U=$ $u-\Phi$. Then the function $U$ satisfies boundary conditions (58). By the variational principle

$$
\left(G_{n, k} U, G_{n, k} v\right)+\alpha[U, v]=\left(H_{n, k}-G_{n, k} \Phi, G_{n, k} v\right)-\alpha[\Phi, v],
$$

for all functions $v \in H^{5}(\Omega)$ satisfying boundary conditions (58). Here

$$
G_{n, k} U:=\Delta U-a_{n, k} \nabla U
$$

The rest of the proof follows from the Riesz theorem.

Lemma 5.3 ([30], see the second paragraph of Introduction). Let the function $u \in H^{5}(\Omega)$ satisfies boundary conditions (58) as well as the variational equality

$$
\left(G_{n, k} u, G_{n, k} v\right)+\alpha[u, v]=\left(H_{n, k}, G_{n, k} v\right)+\alpha[g, v]
$$

for all functions $v \in H^{5}(\Omega)$ satisfying (58), where the operator $G_{n, k}$ is defined in (80). Then

$$
\|u\|_{H^{5}(\Omega)} \leq \alpha^{-1 / 2}\left\|H_{n, k}\right\|_{L_{2}(\Omega)}+\|g\|_{H^{5}(\Omega)} .
$$

Theorem 5.1 ([30], see the second paragraph of Introduction). Assume that condition (79) holds. Let $g \in H^{5}(\Omega)$ be an arbitrary function. Let $u \in H^{5}(\Omega)$ be the function satisfying boundary conditions (58) as well as the variational equality (81) for all functions $v \in H^{5}(\Omega)$ satisfying (58). Let the number $\varkappa \in(1 / 3,1)$ and the number $\rho \in(\varkappa, 1)$. Consider the numbers $b_{1}, b_{2}$,

$$
\begin{array}{r}
b_{1}:=b_{1}(M, \varkappa, \rho)=\frac{1}{2\left(1+\left(1-\rho^{\nu_{0}}\right)(3 \rho)^{-\nu_{0}}\right)} \in\left(0, \frac{1}{2}\right), \\
b_{2}:=b_{2}(M, \varkappa, \rho)=\frac{1}{2}-b_{1}>0,
\end{array}
$$


where $\nu_{0}$ is the parameter of Lemma 5.1. Then there exists a sufficiently small number $\alpha_{1}=\alpha_{1}(M, \varkappa, \rho) \in(0,1)$ such that for all $\alpha \in\left(0, \alpha_{1}\right)$ the following estimate holds with $a$ constant $C_{2}=C_{2}(M, \varkappa, \rho)>0$

$$
\|u\|_{H^{2}\left(\Omega_{\varkappa}\right)} \leq C_{2} \alpha^{-b_{1}}\left\|H_{n, k}\right\|_{L_{2}(\Omega)}+\alpha^{b_{2}}\|g\|_{H^{5}(\Omega)} .
$$

The proof of Theorem 5.1 is based on Lemma 5.1. An important difference between Theorem 2.1 of [16] and Theorem 5.1 is that we now use a new CWF in order to take into account the Neumann boundary condition at $\Gamma_{2}$. Indeed, an analog of our Neumann boundary condition at $\Gamma_{2}$ was not used in [16]. Recall that we need the Neumann boundary condition at $\Gamma_{2}$ only to ensure a better stability of the numerical solution of our target CIP (the fourth Remark 4.1).

\section{Preliminaries for the Convergence Analysis}

We follow the concept of Tikhonov for ill-posed problems [19, 34, 40]. By this concept, one should assume that there exists an "ideal" exact solution of an ill-posed problem with the "ideal" exact data. Next, one should prove that the regularized solution is close to the exact one. In the course of this proof one can use an a priori upper estimate of a certain norm of the exact solution.

\subsection{Exact solution}

First, we need to introduce the definition of the exact solution. Several aspects of this definition are different from ones in previous publications $[4,5,27]$. We assume that there exists a coefficient $c^{*}(x)$ which is the unique exact solution of our Inverse Problem with the exact (i.e. noiseless) data $\bar{\varphi}_{0}^{*}(x, t), \bar{\varphi}_{1}^{*}(x, t)$ in (8) (Remark 2.1). We assume without loss of generality that

$$
\left\|c^{*}\right\|_{C^{\gamma}\left(\mathbb{R}^{3}\right)} \leq d \text { and conditions (3), (4) hold for } c^{*}(x) .
$$

Let $u^{*}(x, t)$ be the solution of the forward problem (1), (2) with $c:=c^{*}$ and $w^{*}(x, s)$ be its Laplace transform (10) for $s>\underline{s}^{0}(d)>0$. Since the source $x_{0} \notin \bar{\Omega}$, then it follows from (84) and Theorem 3.1 that $w^{*}(x, s) \in C^{5+\gamma}(\bar{\Omega})$. Similarly with section 4 let $v^{*}(x, s)=$ $s^{-2} \ln w^{*}(x, s)$ and $q^{*}(x, s)=\partial_{s} v^{*}(x, s), s>\underline{s}(d)$. Let $[\underline{s}, \bar{s}]$ be the $s$-interval of section 4 and $\underline{s}>\underline{s}(d)$. We assume that

$$
q^{*} \in C^{5+\gamma}(\bar{\Omega}) \times C^{1}[\underline{s}, \bar{s}],\left\|q^{*}(x, s)\right\|_{C^{5+\gamma}(\bar{\Omega}) \times C^{1}[\underline{s}, \bar{s}]} \leq C^{*} ; C^{*}=\text { const. }>0,
$$

where $C^{*}$ is the given upper bound of the norm in (85). Consider the same partition of the interval $[\underline{s}, \bar{s}]$ into $N$ small subintervals as in section 4 . Let $q_{n}^{*}(x)$ be the average of the function $q^{*}(x, s)$ over the interval $\left(s_{n}, s_{n-1}\right)$. Then (85) implies that

$$
\max _{s \in\left[s_{n}, s_{n-1}\right]}\left\|q_{n}^{*}(x)-q^{*}(x, s)\right\|_{C^{5+\gamma}(\bar{\Omega})} \leq C^{*} h .
$$


Hence, increasing, if necessary the number $C^{*}$, we can assume that

$$
\max _{1 \leq n \leq N}\left\|q_{n}^{*}\right\|_{H^{5}(\Omega)} \leq C^{*} .
$$

Let

$$
\psi_{0}^{*}(x, s)=\left.q^{*}(x, s)\right|_{\Gamma_{1}}, \psi_{1}^{*}(x, s)=\left.\partial_{z} q^{*}(x, s)\right|_{\Gamma_{1}}, s \in[\underline{s}, \bar{s}] .
$$

For $x \in \Gamma_{1}$ let functions $\psi_{0, n}^{*}(x)$ and $\psi_{1, n}^{*}(x)$ be averages of functions $\psi_{0}^{*}(x, s)$ and $\psi_{1}^{*}(x, s)$ over the interval $\left(s_{n}, s_{n-1}\right)$. Then boundary conditions for functions $q_{n}^{*}(x)$ at $\Gamma_{1}$ are

$$
\left.q_{n}^{*}\right|_{\Gamma_{1}}=\psi_{0, n}^{*}(x),\left.\partial_{z} q_{n}^{*}\right|_{\Gamma_{1}}=\psi_{1, n}^{*}(x) .
$$

The exact tail function $V^{*}(x)$ is

$$
V^{*}(x)=\bar{s}^{-2} \ln w^{*}(x, \bar{s}) .
$$

The function $q_{n}^{*}$ satisfies the following analogue of equation (42)

$$
\begin{aligned}
& \Delta q_{n}^{*}-A_{1, n}\left(h \sum_{j=0}^{n-1} \nabla q_{j}^{*}(x)-\nabla V^{*}\right) \nabla q_{n}^{*}=-A_{2, n} h^{2}\left(\sum_{j=0}^{n-1} \nabla q_{j}^{*}(x)\right)^{2} \\
& +2 A_{2, n} \nabla V^{*}\left(h \sum_{j=0}^{n-1} \nabla q_{j}^{*}(x)\right)-A_{2, n}\left(\nabla V^{*}\right)^{2}+F_{1, n}(x, h, \mu), q_{0}^{*}: \equiv 0 .
\end{aligned}
$$

Similarly with (46) and (47)

$$
\begin{aligned}
& v_{n}^{*}(x):=-h q_{n}^{*}(x)-h \sum_{j=0}^{n-1} q_{j}^{*}(x)+V^{*}(x), x \in \Omega, \\
& c^{*}(x)=\Delta v_{n}^{*}(x)+s_{n}^{2}\left|\nabla v_{n}^{*}(x)\right|^{2}+F_{2, n}(x, h), x \in \Omega .
\end{aligned}
$$

In (91) and (93) functions $F_{1, n}(x, h, \mu), F_{2, n}(x, h)$ represent approximation errors due to averaging and the $s$-dependent Carleman Weight Function. In particular, the term $B_{n}(\mu, h)\left(\nabla q_{n}^{*}\right)^{2}$, which we have ignored in (43) (Remarks 4.1), is a part of $F_{1, n}$. Although we can prove an analog of Theorem 7.1 for the case $F_{1, n} \neq 0, F_{2, n} \neq 0, \psi_{0, n}^{*} \neq \psi_{0, n}, \psi_{1, n}^{*} \neq \psi_{1, n}$, this would require more space. At the same time, the method of the proof would be almost the same. Thus, we will "allow" the error in the boundary data at $\Gamma_{1}$ to be present only at $s:=\bar{s}$, see Lemma 7.1. Therefore, for brevity only we assume below that

$$
F_{1, n}=0, F_{2, n}=0, \psi_{0, n}^{*}=\psi_{0, n}, \psi_{1, n}^{*}=\psi_{1, n}, n \in[1, N] .
$$

Using, ideas of the proof of Theorem 3.1, it is possible to prove that for $s>\underline{s}(d)$ not only the function $w^{*}(x, s) \in C^{5+\gamma}(\bar{\Omega})$ but also functions $D_{s}^{k} w^{*}(x, s) \in C^{5+\gamma}(\bar{\Omega}), k=1,2$. Since this implies that $q^{*}(x, s) \in C^{5+\gamma}(\bar{\Omega}) \times C^{1}[\underline{s}, \bar{s}]$, then it is not necessary to impose this assumption in (85). However, we still prefer to use this assumption in order to save space and also because this is not our main focus. The reason why we require the $C^{4}-$ smoothness of $c^{*}$ in (4) is to ensure that $V^{*} \in C^{5+\gamma}(\bar{\Omega})$. We need the latter to justify the assumption in $(97)$ that $p^{*} \in H^{5}(\Omega)$. 


\subsection{Approximate global convergence}

Definition 6.1 (approximate global convergence). Consider a nonlinear ill-posed problem. Suppose that a certain approximate mathematical model $M$ is proposed to solve this problem numerically. Assume that, within the framework of the model $M$, this problem has unique exact solution $x^{*} \in B$ for the noiseless data $y^{*}$. Here $B$ is an appropriate Banach space with the norm $\|\cdot\|_{B}$. Consider an iterative numerical method for solving that problem. Suppose that this method produces a sequence of points $\left\{x_{n}\right\}_{n=1}^{K} \subset B, K \in[1, \infty)$. Let the number $\theta \in(0,1)$. We call this numerical method approximately globally convergent of the level $\theta$ in the norm of the space $B$, or shortly globally convergent, if, within the framework of the model $M$, a theorem is proven, which claims that, without any knowledge of a sufficiently small neighborhood of $x^{*}$, there exists a number $\bar{K} \in[1, K)$ such that the following inequality is valid

$$
\frac{\left\|x_{n}-x^{*}\right\|_{B}}{\left\|x_{n}\right\|_{B}} \leq \theta, \forall n \geq \bar{K}
$$

Suppose that iterations are stopped at a certain number $k \geq \bar{K}$. Then the point $x_{k}$ is denoted as $x_{k}:=x_{g l o b}$ and is called the approximate solution resulting from this method.

This is our formal mathematical definition of the approximate global convergence property. However, since the approximate mathematical model $M$ is involved in it, then it is reasonable to raise the question about a verification of $M$. It is natural to address this question via computational testing. Hence, the following two informal conditions should be added to Definition 6.1:

A. Numerical studies should confirm that $x_{g l o b}$ is indeed a sufficiently good approximation for the exact solution $x^{*}$.

B (optional). Testing of this numerical method on appropriate experimental data also demonstrates that iterative solutions provide a good approximation for the exact one.

We consider condition $\mathbf{B}$ as an optional one because, in our experience, it is often both hard and expensive to obtain proper experimental data. Furthermore, these data might be suitable only for one version of that numerical method and not suitable for other versions. Definition 6.1 is worthy of some discussion, which is done below in this subsection.

The single reason why we have introduced Definition 6.1 is a substantial challenge in the goal of constructing such a numerical method for a CIP which would provide a good approximation for the exact solution without an advanced knowledge of a small neighborhood of this solution (section 1). Because of this challenge, it is unlikely that the desired good approximation for the exact solution would be obtained without some approximations. Thus, we use the approximate mathematical model $M$.

The main requirement of Definition 6.1 is that this numerical method should provide a sufficiently good approximation for the exact solution $x^{*}$, regardless on any a priori knowledge of a sufficiently small neighborhood of $x^{*}$. Furthermore, it is important that one should have a rigorous guarantee of the latter, again within the framework of the model $M$. Unlike the classical convergence, this definition does not require that $\lim _{n \rightarrow \infty} x_{n}=x^{*}$. Furthermore, the total number of iterations can be finite and should be chosen as a result of numerical 
studies. We remind that it is quite often in the field of Ill-Posed Problems that the number of iterations is a regularization parameter, see, e.g. pages 156 and 157 of [19].

Therefore, Definition 6.1 leaves the room for a refinement of the approximate solution $x_{g l o b}$ via a subsequent application of a locally convergent numerical method. In other words, the room is left for a two-stage numerical procedure $[5,6,7,8]$. In accordance with the theory of Ill-Posed Problems [19, 34, 40], some a priori knowledge about the exact solution $x^{*}$ should still be in place of course, see, e.g. (3) and (84)-(87).

Remark 6.1. As to the approximate mathematical model $M$, here is a good analogy. It is well known that the Huygens-Fresnel optics is not yet rigorously derived from the Maxwell equations. We now cite some relevant statements from section 8.1 of the classical book of Born and Wolf [12]. First, "Diffraction problems are amongst the most difficult ones encountered in optics. Solutions which, in some sense, can be regarded as rigorous are very rare in diffraction theory." Next, "because of mathematical difficulties, approximate models must be used in most cases of practical interest. Of these the theory of Huygens and Fresnel is by far the most powerful and is adequate for the treatment of the majority of problems encountered in instrumental optics." It is well known that the entire optical industry nowadays is based on the Huygens-Fresnel theory. Analoguosly, although our method of both the current and the above cited previous publications works only with approximate models, its accurate numerical performance has been consistently demonstrated in the above cited publications.

\subsection{Our approximate mathematical model}

Assuming that conditions of Lemma 3.1 hold, Assumptions 1-3 below mean that we take into account only the first term of the asymptotic behavior of the function $s^{-2} \ln w^{*}(x, s)$ at $s \rightarrow \infty$ and ignore the rest. Assumption 4 means that we assume that the function $q^{*}(x, s)$ satisfies the third boundary condition (42). Recall that the latter condition is an approximate one.

The equation for the function $q^{*}$ is (see formula (85) in [4])

$$
\begin{aligned}
& \Delta q^{*}-2 s^{2} \nabla q^{*} \int_{s}^{\bar{s}} \nabla q^{*}(x, \tau) d \tau+2 s\left[\int_{s}^{\bar{s}} \nabla q^{*}(x, \tau) d \tau\right]^{2}+2 s^{2} \nabla q^{*} \nabla V^{*} \\
& -2 s \nabla V^{*} \int_{s}^{\bar{s}} \nabla q^{*}(x, \tau) d \tau+2 s\left(\nabla V^{*}\right)^{2}=0,(x, s) \in \Omega \times(\underline{s}, \bar{s}) .
\end{aligned}
$$

Our approximate mathematical model $M$ consists of the following four assumptions:

Assumptions. We assume below that:

1. The exact solution of our Inverse Problem $c^{*}(x)$ exists and satisfies condition (84). 
2. There exists a function $p^{*}(x) \in H^{5}(\Omega)$ such that the exact tail function $V^{*}(x)$ has the form (see (90))

$$
V^{*}(x, \bar{s}):=V^{*}(x)=\frac{p^{*}(x)}{\bar{s}}=\frac{\ln w^{*}(x, \bar{s})}{\bar{s}^{2}} .
$$

3. For $\bar{s}>\underline{s}>\underline{s}^{0}(d)>0$ and for the tail function $V^{*}(x)$ in the form (97) the function $q^{*}(x, s),(x, s) \in \Omega \times[\underline{s}, \bar{s}]$ satisfies condition (85), equation (96). In addition for $s:=\bar{s}$ the function $q^{*}(x, \bar{s})$ has the form

$$
q^{*}(x, \bar{s})=-\frac{p^{*}(x)}{\bar{s}^{2}} .
$$

4. For $s \in[\underline{s}, \bar{s}]$ the function $q^{*}(x, s)$ satisfies boundary conditions (88) at $\Gamma_{1}$ as well as the third boundary condition (42) at $\Gamma_{2}$

$$
\left.\partial_{n} q^{*}(x, s)\right|_{\Gamma_{2}}=s^{-2}, \forall s \in[\underline{s}, \bar{s}] .
$$

In addition for $s:=\bar{s}$ the function $q^{*}(x, \bar{s})$ satisfies the third boundary condition $(42)$ at $\Gamma_{3}$

$$
\left.\partial_{z} q^{*}(x, \bar{s})\right|_{\Gamma_{3}}=\bar{s}^{-2} .
$$

It follows from (99) that

$$
\left.\partial_{n} q_{n}^{*}\right|_{\Gamma_{2}}=\left(s_{n} s_{n-1}\right)^{-1} .
$$

Set in (96) $s=\bar{s}$. Then, using (88) and (97)-(100), we obtain the following approximate $\mathrm{PDE}$ and boundary conditions for the function $p^{*}(x)$

$$
\begin{aligned}
\Delta p^{*} & =0 \text { in } \Omega, p^{*} \in H^{5}(\Omega), \\
\left.p^{*}\right|_{\Gamma_{1}} & =-\bar{s}^{2} \psi_{0}^{*}(x, \bar{s}),\left.\partial_{n} p^{*}\right|_{\Gamma_{1}}=-\bar{s}^{2} \psi_{1}^{*}(x, \bar{s}),\left.\partial_{n} p^{*}\right|_{\Gamma_{2} \cup \Gamma_{3}}=-1 .
\end{aligned}
$$

Boundary conditions (103) are over-determined ones. The existence of the solution of the problem (102), (103) is actually assumed because conditions (102), (103) are derived from Assumptions 1-4. Let functions $\psi_{0}, \psi_{1}$ be the boundary conditions in (42). Suppose that for each $\alpha \in(0,1)$ there exists the QRM solution $p=p(x ; \alpha)$ of the following boundary value problem

$$
\begin{aligned}
\Delta p & =0 \text { in } \Omega, p(x) \in H^{5}(\Omega), \\
\left.p\right|_{\Gamma_{1}} & =-\bar{s}^{2} \psi_{0}(x, \bar{s}),\left.\partial_{n} p\right|_{\Gamma_{1}}=-\bar{s}^{2} \psi_{1}(x, \bar{s}),\left.\partial_{n} p\right|_{\Gamma_{2} \cup \Gamma_{3}}=-1,
\end{aligned}
$$

see Lemma 7.1 for the existence and uniqueness of the function $p$. Then we choose an appropriate $\alpha \in(0,1)$ and in the iterative process of subsection 4.3 we set the first approximation for the tail function as

$$
V_{1,1}(x):=V_{1,1}(x ; \alpha):=\frac{p(x ; \alpha)}{\bar{s}} .
$$

To show that equation (102) is a reasonable approximation, consider, for example, the simplest case $c(x) \equiv 1$. Then by (14) and (90) $p^{*}(x)=-\left|x-x_{0}\right|$. Hence,

$$
\Delta p^{*}=-\left|x-x_{0}\right|^{-1} \approx 0 \text { in } \Omega,
$$


as long as the source $x_{0}$ is located far from the domain $\Omega$, as it is the case in many applications. In the more general case of the asymptotics $(36) p^{*}(x)=l\left(x, x_{0}\right) \geq\left|x-x_{0}\right|$. Hence, by an analogy with (107), assume that $\left|\Delta l\left(x, x_{0}\right)\right| \leq \bar{C} l^{-1}\left(x, x_{0}\right), x \in \Omega$, where $\bar{C}>0$ is a certain constant independent on $x, x_{0}$. Since $l\left(x, x_{0}\right) \geq\left|x-x_{0}\right|$, then $\left|\Delta l\left(x, x_{0}\right)\right| \leq$ $\bar{C}\left|x-x_{0}\right|^{-1}, x \in \Omega$. Hence, if the source $x_{0}$ is located far from $\Omega$, then similarly with (107) $\Delta l\left(x, x_{0}\right) \approx 0$ in $\Omega$. The function $l\left(x, x_{0}\right)$ satisfies the eikonal equation [38] $\left|\nabla_{x} l\left(x, x_{0}\right)\right|^{2}=$ $c(x)$. However, one cannot consider this equation in our case, since its right hand side is unknown. There is of course a well known the so-called "inverse kinematic problem" which aims to recover the function $c(x)$ from the eikonal equation assuming that the function $l\left(x, x_{0}\right)$ is known for all $x, x_{0} \in \partial \Omega$ [38]. However, we have only one source position here. Consider now the case when the incident plane wave is originated at the plane $\left\{x_{3}=x_{30}\right\}$. The function $w$ corresponding to the case $c(x) \equiv 1$ is $w=\exp \left(-s\left|x_{3}-x_{3,0}\right|\right) /(2 s)$. Hence, if $\bar{\Omega} \subset\left\{x_{3}>x_{3,0}\right\}$ and $c(x) \equiv 1$, then $\Delta p^{*}(x)=-\Delta\left(x_{3}-x_{3,0}\right)=0$ in $\Omega$, which is the same as $(102)$.

We now establish uniqueness within the framework of our approximate mathematical model. Although it can be proven under less restrictive assumptions imposed on functions $q^{*}, p^{*}$ than ones above, we are not doing this here for brevity.

Lemma 6.1. Suppose that above assumptions 1-4 hold. Then there exists at most one function $q^{*}(x, s)$ for $(x, s) \in \Omega \times[\underline{s}, \bar{s}]$. In addition, if assuming the continuous analog of (93) (as in [4, 5]) $c^{*}(x)=\Delta v^{*}(x)+s^{2}\left|\nabla v^{*}(x)\right|^{2},(x, s) \in \Omega \times[\underline{s}, \bar{s}]$, where the function $v$ is the same as in (39) with $(q, V):=\left(q^{*}, V^{*}\right)$, then there exists at most one function $c^{*}(x)$.

Proof. We outline the proof only briefly because it is simple. Uniqueness of the problem (102), (103) is obvious. Next, substituting in (96) the function $V^{*}(x)$ from (97) and applying the Carleman estimate of Lemma 5.1 to (96) we quickly obtain uniqueness of the function $q^{*}(x, s)$. The $s$-integrals are not a problem in this case since the Carleman estimate is independent on lower order derivatives and it can be integrated with respect to $s \in(\underline{s}, \bar{s})$.

\subsection{Estimates for the tail function}

Below in sections $6,7 B=B\left(\bar{s}, d, x_{0}\right)>2$ denotes different constants depending on listed parameters. We do not indicate its dependence on the domain $\Omega$, because $\Omega$ is as in (57) in these sections.

Theorem 6.1. Let the domain $\Omega$ be as in (57) and the source $x_{0} \notin \bar{\Omega}$. Let the function $c^{*}(x)$ satisfying (84) be the exact solution of our inverse problem as defined in subsection 6.1. Let $0<\underline{s}(d)<\underline{s}<\bar{s}$ and $V^{*}(x)$ be the exact tail function as in (90). For each function $c(x)$ satisfying condition (9) and for $s \in[\underline{s}, \bar{s}]$ let $w(x, s ; c):=w(x, s)$ be the solution of the problem (11), (12) (Theorem 3.1). Denote $V(x ; c):=V(x):=\bar{s}^{-2} \ln w(x, \bar{s} ; c)$. Then with a constant $B=B\left(\bar{s}, d, x_{0}\right)$

$$
\begin{aligned}
\|\nabla V\|_{C(\bar{\Omega})},\left\|\nabla V^{*}\right\|_{C(\bar{\Omega})} & \leq B \\
\left\|\nabla V-\nabla V^{*}\right\|_{L_{2}(\Omega)}+\left\|\Delta V-\Delta V^{*}\right\|_{L_{2}(\Omega)} & \leq B\left\|c-c^{*}\right\|_{L_{2}(\Omega)},
\end{aligned}
$$


Proof. Recall that $x=(x, y, z)$. For brevity we estimate only $\left\|V_{x}\right\|_{C(\bar{\Omega})}$. Estimates of two other first derivatives are similar. By (16) and (90)

$$
\left|V_{x}\right|=\left|\frac{w_{x}}{w}(x, \bar{s})\right| \leq B\left|w_{x}(x, \bar{s})\right|,\left|V_{x}^{*}\right|=\left|\frac{w_{x}^{*}}{w^{*}}(x, \bar{s})\right| \leq B\left|w_{x}^{*}(x, \bar{s})\right| .
$$

Theorem 3.1, (14) and (30) imply that for $\xi=\left(\xi_{1}, \xi_{2}, \xi_{3}\right), x \in \Omega, b(x)=c(x)-1$

$$
\begin{aligned}
w_{x}(x, \bar{s})= & w_{0 x}(x, \bar{s})+ \\
& \frac{s^{2}}{4 \pi} \int_{\Omega}\left[\left(s \frac{x-\xi_{1}}{|x-\xi|^{2}}+\frac{x-\xi_{1}}{|x-\xi|^{3}}\right) \exp (-\bar{s}|x-\xi|) b(\xi) w(\xi, \bar{s})\right] d \xi .
\end{aligned}
$$

Since $x_{0} \notin \bar{\Omega}$, then functions $w_{0}, w_{0 x}$ do not have a singularity for $x \in \bar{\Omega}$. Hence, (16) and (111) imply that

$$
\left|w_{x}(x, \bar{s})\right| \leq B+B \int_{\Omega}\left(\bar{s} \frac{1}{|x-\xi|}+\frac{1}{|x-\xi|^{2}}\right) \exp (-\bar{s}|x-\xi|) d \xi \leq B, x \in \Omega .
$$

Hence, (108) follows from (110) and (112). Next, for $\widetilde{w}:=w-w^{*}$

$$
V_{x}-V_{x}^{*}=\left(\frac{\widetilde{w}_{x}}{w}-\frac{w_{x}^{*}}{w w^{*}} \widetilde{w}\right)(x, \bar{s}), x \in \Omega .
$$

Hence, by (16) and (112)

$$
\begin{aligned}
\left\|\nabla V-\nabla V^{*}\right\|_{L_{2}(\Omega)} \leq & B\left(\|\nabla \widetilde{w}\|_{L_{2}(\Omega)}+\|\widetilde{w}\|_{L_{2}(\Omega)}\right) \leq \\
& B\left(\|\nabla \widetilde{w}\|_{L_{2}\left(\mathbb{R}^{3}\right)}+\|\widetilde{w}\|_{L_{2}\left(\mathbb{R}^{3}\right)}\right) .
\end{aligned}
$$

Let $\widetilde{c}=c-c^{*}$. Since $c w-c^{*} w^{*}=c \widetilde{w}+\widetilde{c} w^{*}$, we obtain from (11)

$$
\Delta \widetilde{w}-\bar{s}^{2} c(x) \widetilde{w}=\bar{s}^{2} \widetilde{c} w^{*}, x \in \mathbb{R}^{3} .
$$

Let the number $R>0$ be so large that $\Omega \subset B_{R}=\{|x|<R\}$. Multiply both sides of (114) by $(-\widetilde{w})$ and integrate over $B_{R}$. Since $\widetilde{c}(x)=0$ outside of $\Omega$, we obtain

$$
\begin{aligned}
\int_{B_{R}}\left(|\nabla \widetilde{w}|^{2}+\bar{s}^{2} c \widetilde{w}^{2}\right)(x, \bar{s}) d x-\int_{\partial B_{R}}\left(\widetilde{w} \frac{\partial \widetilde{w}}{\partial n}\right)(x, \bar{s}) d S= & \\
& -\bar{s}^{2} \int_{\Omega}\left(\widetilde{c} w^{*} \widetilde{w}\right)(x, \bar{s}) d x .
\end{aligned}
$$

It follows from $(30)$ and $(111)$ that $\nabla \widetilde{w}(x, \bar{s}), \widetilde{w}(x, \bar{s}) \in L_{2}\left(\mathbb{R}^{3}\right)$ and the second term in the left hand side of (115) tends to zero as $R \rightarrow \infty$. Hence, setting in (115) $R \rightarrow \infty$, we obtain

$$
\int_{\mathbb{R}^{3}}\left(|\nabla \widetilde{w}|^{2}+\bar{s}^{2} c \widetilde{w}^{2}\right)(x, \bar{s}) d x=-\bar{s}^{2} \int_{\Omega}\left(\widetilde{c} w^{*} \widetilde{w}\right)(x, \bar{s}) d x .
$$


Since $c \geq 1$, then $\bar{s}^{2} c \widetilde{w}^{2}(x, \bar{s}) \geq \bar{s}^{2} \widetilde{w}^{2}(x, \bar{s})$. Hence, using (16) and the Cauchy-Schwarz inequality, we obtain

$$
\|\widetilde{w}(x, \bar{s})\|_{H^{1}\left(\mathbb{R}^{3}\right)} \leq B\|\widetilde{c}\|_{L_{2}(\Omega)} .
$$

Next,

$$
\begin{aligned}
\Delta V-\Delta V^{*} & = \\
& =\left[\frac{\Delta \widetilde{w}}{w}-\frac{\nabla\left(w+w^{*}\right)}{w^{2}} \nabla \widetilde{w}-\left(\frac{\Delta w^{*}}{w w^{*}}-\frac{\left(\nabla w^{*}\right)^{2}\left(w+w^{*}\right)}{\left(w w^{*}\right)^{2}}\right) \widetilde{w}\right](x, \bar{s})
\end{aligned}
$$

Since $\Delta w^{*}(x, \bar{s})=\bar{s}^{2}\left(c^{*} w^{*}\right)(x, \bar{s})$ for $x \in \bar{\Omega}$, then (16), (112) and (117) imply that

$$
\left|\Delta V-\Delta V^{*}\right| \leq B(|\Delta \widetilde{w}|+|\nabla \widetilde{w}|+|\widetilde{w}|), x \in \bar{\Omega} .
$$

By (16) and (114) $\|\Delta \widetilde{w}\|_{L_{2}\left(\mathbb{R}^{3}\right)} \leq B\left(\|\widetilde{w}\|_{L_{2}\left(\mathbb{R}^{3}\right)}+\|\widetilde{c}\|_{L_{2}(\Omega)}\right)$. Hence, (113), (116) and (118) imply (109).

\section{Approximate Global Convergence Theorem}

Assume that

$$
\bar{s}>1, \mu h \geq 1
$$

Then [4]

$$
\max _{1 \leq n \leq N}\left\{\left|A_{1, n}\right|+\left|A_{2, n}\right|\right\} \leq 8 \bar{s}^{2} .
$$

In general, embedding theorems are valid for domains with sufficiently smooth boundaries. It follows from Lemma 1 of $\S 4$ of Chapter 3 of the book [35] that if $Q$ is a rectangular prism, then any function $f \in H^{k}(Q)$ can be extended in a bigger rectangular prism $Q_{1}$ $\supset Q, \partial Q \cap \partial Q_{1}=\varnothing$ as the function $f_{1} \in H^{k}\left(Q_{1}\right), f_{1}(x)=f(x)$ in $Q$ and $\left\|f_{1}\right\|_{H^{k}\left(Q_{1}\right)} \leq$ $Z\|f\|_{H^{k}(Q)}$, where the constant $Z=Z\left(Q, Q_{1}\right)>0$. Hence, embedding theorems are valid for rectangular prisms. Hence,

$$
\|f\|_{C^{3}(\bar{\Omega})} \leq C\|f\|_{H^{5}(\Omega)}, \forall f \in H^{5}(\Omega)
$$

Let the domain $\Omega$ be the same as in section 5 . Recall that $\Omega_{\varkappa} \subset \Omega$ for $\varkappa \in(1 / 3,1)$ and $\Omega_{1}=\Omega$. Following the construction in the end of subsection 4.1, we assume that

$$
P_{0}:=\varkappa_{0} \in(1 / 3,1), c(x)=1 \text { for } x \in \mathbb{R}^{3} \backslash \Omega_{\varkappa_{0}} .
$$

Since $\widehat{c}_{n, k}(x) \neq c_{n, k}(x)$ for $x \in \Omega_{\varkappa_{0}} \backslash \Omega^{\prime}$, then the number meas $\left(\Omega_{\varkappa_{0}} \backslash \Omega^{\prime}\right)$ can be considered as a part of the error in the data. Hence, we assume that $\Omega^{\prime}$ is such that

$$
\operatorname{meas}\left(\Omega_{\varkappa_{0}} \backslash \Omega^{\prime}\right)<\varepsilon / 2 \text {, }
$$


where $\varepsilon \in(0,1)$ is sufficiently small. Since by construction $\widehat{c}_{n, k}(x), c^{*}(x) \in[1, d], \forall x \in \mathbb{R}^{3}$ and $\widehat{c}_{n, k}(x)=c_{n, k}(x), \forall x \in \Omega^{\prime}$, then by (48), (122) and (123)

$$
\left\|\widehat{c}_{n, k}-c^{*}\right\|_{L_{2}(\Omega)} \leq\left\|c_{n, k}-c^{*}\right\|_{L_{2}\left(\Omega^{\prime}\right)}+d \varepsilon \leq\left\|\bar{c}_{n, k}-c^{*}\right\|_{L_{2}\left(\Omega^{\prime}\right)}+d \varepsilon .
$$

As it is always the case in the convergence analysis of ill-posed problems, we need to connect the regularization parameter $\alpha$ of the QRM in (56) with various approximation errors: the level of the error $\sigma$ in the data (Lemma 7.1), the grid step size $h$ in the $s$-direction and the number $\varepsilon$ in (123). In addition, to iteratively "suppress" the large parameter $\alpha^{-b_{1}}$ in (83) (to ensure the stability of our process), we need to impose a smallness assumption on the length $\beta=\bar{s}-\underline{s}=N h$, where $N \geq 1$ is an integer. For a number $x>0$ let $\{x\}^{\circ}$ denotes such an integer that $x-\{x\}^{\circ} \in[0,1)$. Thus, we impose the following conditions

$$
\begin{aligned}
\sigma, \varepsilon & \in(0, \sqrt{\alpha}], \\
h & =\sqrt{\alpha}, \beta:=\beta(\alpha)=\sqrt{\alpha}\{f(\alpha)\}^{\circ}:=\sqrt{\alpha} N,
\end{aligned}
$$

where the function $f(\alpha)$ is monotonically decreasing for $\alpha \in(0,1)$,

$$
f(\alpha)>0 \text { for } \alpha \in(0,1), \lim _{\alpha \rightarrow 0^{+}} f(\alpha)=\infty \text { and } \lim _{\alpha \rightarrow 0^{+}} \frac{f(\alpha)}{\ln \left(\alpha^{-1}\right)}=0 .
$$

Two examples of the function $f(\alpha)$ are $f_{1}(\alpha)=\left[\ln \left(\alpha^{-1}\right)\right]^{r}, r=$ const. $\in(0,1)$ and $f_{2}(\alpha)=$ $\ln \left(\ln \left(\alpha^{-1}\right)\right)$.

Let $\left(q_{n, k}, \bar{c}_{n, k}, V_{n, k}\right)$ be the triple computed on a certain step of our iterative process of subsection 4.2. Denote

$$
\widetilde{q}_{n, k}=q_{n, k}-q_{n}^{*}, \widetilde{c}_{n, k}=\bar{c}_{n, k}-c^{*}, \widetilde{V}_{n, k}=V_{n, k}-V^{*} .
$$

Similarly for $\widetilde{q}_{n}, \widetilde{c}_{n}, \widetilde{V}_{n}$. Even though we have assumed (for brevity only) that there is no error in functions of (94), Lemma 7.1 and Theorem 7.1 "allow" error to be present in functions $\psi_{0}^{*}(x, \bar{s}), \psi_{1}^{*}(x, \bar{s})$ in $(103)$, see the first two sentences of the proof of Lemma 7.1.

Lemma 7.1 (estimate of $\widetilde{V}_{1,1}$ ). Let the domain $\Omega$ be as in (57) and the source $x_{0} \notin \bar{\Omega}$. Let Assumptions 1-4 of subsection 6.3 hold as well as (125). Let $\Psi^{*} \in H^{5}(\Omega)$ be a function satisfying boundary conditions (103). Suppose that there exists a function $\Psi \in H^{5}(\Omega)$ satisfying boundary conditions (105). Let the number $\sigma \in(0,1)$ be the level of the error in the function $\Psi^{*}$ when it is replaced with the function $\Psi$ and

$$
\begin{aligned}
\left\|\Psi-\Psi^{*}\right\|_{H^{5}(\Omega)} & \leq \sigma \leq \sqrt{\alpha} \\
\left\|p^{*}\right\|_{H^{5}(\Omega)} & \leq B .
\end{aligned}
$$

Let the function $p=p(x ; \alpha) \in H^{5}(\Omega)$ be the unique QRM solution of the problem (104), (105) which is guaranteed by Lemma 5.2, and the tail function $V_{1,1}(x):=V_{1,1}(x ; \alpha)$ has the form (106). Then for every $\alpha \in(0,1)$

$$
\begin{aligned}
\left\|\nabla \widetilde{V}_{1,1}\right\|_{L_{2}(\Omega)}+\left\|\Delta \widetilde{V}_{1,1}\right\|_{L_{2}(\Omega)} & \leq B \sqrt{\alpha} \\
\left\|\nabla V_{1,1}\right\|_{C(\bar{\Omega})} & \leq B
\end{aligned}
$$


Proof. Note that the trace theorem, (128), (103) and (105) imply that

$$
\left\|\psi_{0}^{*}(x, \bar{s})-\psi_{0}(x, \bar{s})\right\|_{H^{1}\left(\Gamma_{1}\right)}+\left\|\psi_{1}^{*}(x, \bar{s})-\psi_{0}(x, \bar{s})\right\|_{L_{2}\left(\Gamma_{1}\right)} \leq C \sigma / \bar{s}^{2} .
$$

This means that the error is introduced in the boundary data $\psi_{0}^{*}(x, \bar{s}), \psi_{1}^{*}(x, \bar{s})$ and its level is proportional to $\sigma \in(0, \sqrt{\alpha}]$. For brevity we do not put in this proof the dependence of the function $p$ on $\alpha$. The existence of the function $\Psi^{*}$ follows from Assumptions 1-4. Denote $\widetilde{p}(x)=(p-\Psi)(x)-\left(p^{*}-\Psi^{*}\right)(x)$. Then

$$
(\Delta \widetilde{p}, \Delta v)+\alpha[\widetilde{p}, v]=\left(\Delta \Psi^{*}-\Delta \Psi, \Delta v\right)+\alpha\left[\Psi^{*}-\Psi, v\right]+\alpha\left[p^{*}, v\right]
$$

for all functions $v \in H^{5}(\Omega)$ satisfying zero boundary conditions (105). Setting here $v:=\widetilde{p}$, and using (125), (128), and (129), we obtain

$$
\|\Delta \widetilde{p}\|_{L_{2}(\Omega)}^{2}+\alpha\|\widetilde{p}\|_{H^{5}(\Omega)}^{2} \leq \alpha B^{2} .
$$

Estimate $\|\Delta \widetilde{p}\|_{L_{2}(\Omega)}^{2}$ in (132) from the below. We have

$$
\begin{gathered}
(\Delta \widetilde{p})^{2}=\left(\widetilde{p}_{x x}^{2}+\widetilde{p}_{y y}^{2}+\widetilde{p}_{z z}^{2}\right)+2 \widetilde{p}_{x x} \widetilde{p}_{y y}+2 \widetilde{p}_{x x} \widetilde{p}_{z z}+2 \widetilde{p}_{y y} \widetilde{p}_{z z}, \\
2 \widetilde{p}_{x x} \widetilde{p}_{y y}=\partial_{x}\left(2 \widetilde{p}_{x} \widetilde{p}_{y y}\right)-2 \widetilde{p}_{x} \widetilde{p}_{y y x}=\partial_{x}\left(2 \widetilde{p}_{x} \widetilde{p}_{y y}\right)+\partial_{y}\left(-2 \widetilde{p}_{x} \widetilde{p}_{x y}\right)+2 \widetilde{p}_{x y}^{2}, \\
2 \widetilde{p}_{x x} \widetilde{p}_{z z}=\partial_{x}\left(2 \widetilde{p}_{x} \widetilde{p}_{z z}\right)-2 \widetilde{p}_{x} \widetilde{p}_{z z x}=\partial_{x}\left(2 \widetilde{p}_{x} \widetilde{p}_{z z}\right)+\partial_{z}\left(-2 \widetilde{p}_{x} \widetilde{p}_{x z}\right)+2 \widetilde{p}_{x z}^{2}
\end{gathered}
$$

and similarly for $2 \widetilde{p}_{y y} \widetilde{p}_{z z}$. Integrate (133) over $\Omega$ using these formulas for products. Since by (103) and (105) $\left.\partial_{n} \widetilde{p}\right|_{\partial \Omega}=0$, then boundary integrals will be equal to zero. Next, use

$$
\widetilde{p}_{x}(x, y, z)=\int_{-1 / 4}^{x} \widetilde{p}_{x x}(\xi, y, z) d \xi
$$

and similar formulas for $\widetilde{p}_{y}, \widetilde{p}_{z}$. Using (132), we obtain

$$
\alpha B^{2} \geq\|\Delta \widetilde{p}\|_{L_{2}(\Omega)}^{2} \geq \sum_{|\alpha|=2}\left\|D^{\alpha} \widetilde{p}\right\|_{L_{2}(\Omega)}^{2} \geq C\|\nabla \widetilde{p}\|_{L_{2}(\Omega)}^{2} .
$$

This, (97), (106), (128) and (129) imply (130). Next, by (121), (125), (128) (129) and (132) $\|\nabla p\|_{C(\bar{\Omega})} \leq C\|p\|_{H^{5}(\Omega)} \leq B$. The latter and (106) imply (131)

Theorem 7.1 (approximate global convergence property of our algorithm). Let conditions of Lemma 7.1 hold and $\bar{s}>1$. Also, assume that the following conditions hold: (87)-(94), (119), (122),(123) and (125)-(129). Let the number $\rho \in\left(\varkappa_{0}, 1\right), m$ be the number of inner iterations for functions $q_{n, k}, k \in[1, m]$ (section 4 ) and $f$ be the function in (126), (127). Then there exists a constant $D=D\left(\bar{s}, d, x_{0}, C^{*}, \varkappa_{0}, \rho\right)>1$, numbers 
$b_{1}=b_{1}\left(\bar{s}, d, x_{0}, C^{*}, \varkappa_{0}, \rho\right) \in(0,1 / 2), b_{2}=1 / 2-b_{1}$ defined in Theorem 5.1 and a sufficiently small $\alpha_{0}=\alpha_{0}\left(\bar{s}, d, x_{0}, C^{*}, \varkappa_{0}, \rho, f, m\right) \in(0,1)$ such that the following estimates are valid

$$
\frac{\left\|c_{n}-c^{*}\right\|_{L_{2}\left(\Omega^{\prime}\right)}}{\left\|c_{n}\right\|_{L_{2}\left(\Omega^{\prime}\right)}} \leq \alpha^{b_{2} / 2}, \forall(n, \alpha) \in[1, N] \times\left(0, \alpha_{0}\right) .
$$

Thus, the iterative process of section 4 is approximately globally convergent of the level $\alpha^{b_{2} / 2}$ in the norm of the space $L_{2}\left(\Omega^{\prime}\right)$ (Definition 6.1).

Proof. Theorem 3.1 and (48)-(51) guarantee the existence and uniqueness of tails $V_{n, k}$. Note that because of (85) and (86), the estimate (87) is not changing when the number $N$ of subintervals of the interval $[\underline{s}, \bar{s}]$ increases with the decrease of the parameter $\alpha$. Let $(n, k) \in[1, N] \times[1, m]$. Assuming that the desired constant $D$ is found, we first estimate $D^{2 n m+4} \alpha^{b_{2}}$. Using (126) and (127), we obtain for a sufficiently small number $\alpha_{0}=\alpha_{0}\left(\bar{s}, d, x_{0}, C^{*}, \varkappa_{0}, \rho, f, m\right) \in(0,1)$ and for all $\alpha \in\left(0, \alpha_{0}\right)$

$$
\begin{aligned}
D^{2 n m+4} \alpha^{b_{2}} & \leq D^{2 N m+4} \alpha^{b_{2}} \leq \\
D^{4} \exp \left\{-\ln \left(\alpha^{-1}\right)\left[b_{2}-(2 m \ln D)\left(f(\alpha) / \ln \left(\alpha^{-1}\right)\right)\right]\right\} & <\alpha^{b_{2} / 2} .
\end{aligned}
$$

Below in this proof $\alpha \in\left(0, \alpha_{0}\right)$. Since by $(48) c_{n, k}(x) \geq 1$ in $\Omega_{\varkappa_{0}}$, then $\left\|c_{n, k}\right\|_{L_{2}\left(\Omega^{\prime}\right)} \geq$ meas $\left(\Omega^{\prime}\right)$. Hence, (135) implies that it is sufficient to prove that

$$
\left\|c_{n}-c^{*}\right\|_{L_{2}\left(\Omega^{\prime}\right)} \leq D^{2 n m} \alpha^{b_{2}}, \forall(n, \alpha) \in[1, N] \times\left(0, \alpha_{0}\right) .
$$

By (43), (91), (94) and (101) the function $\widetilde{q}_{n, k}$ is the QRM solution of the following problem

$$
\begin{aligned}
& \Delta \widetilde{q}_{n, k}-A_{1, n}\left(\chi_{2}(x) h \sum_{j=0}^{n-1} \nabla q_{j}-\nabla V_{n, k}\right) \nabla \widetilde{q}_{n, k}=\widetilde{H}_{n, k} \\
&\left.\widetilde{q}_{n, k}\right|_{\Gamma_{1}}=\left.\partial_{z} \widetilde{q}_{n, k}\right|_{\Gamma_{1}}=\left.\partial_{n} \widetilde{q}_{n, k}\right|_{\Gamma_{2}}=0 \\
& \widetilde{H}_{n, k}(x)=-A_{1, n}\left(\chi_{2}(x) h \sum_{j=0}^{n-1} \nabla \widetilde{q}_{j}-\nabla \widetilde{V}_{n, k}\right) \nabla q_{n}^{*} \\
&-A_{2, n}\left(\chi_{2}(x) h \sum_{j=0}^{n-1} \nabla \widetilde{q}_{j}\right)\left(h \sum_{j=0}^{n-1}\left(\nabla q_{j}+\nabla q_{j}^{*}\right)-2 \nabla V_{n, k}\right) \\
&+A_{2, n} \nabla \widetilde{V}_{n, k}\left(2 \chi_{2}(x) h \sum_{j=0}^{n-1} \nabla q_{j}^{*}-\left(\nabla V_{n, k}+\nabla V^{*}\right)\right) \\
&-\left(1-\chi_{2}(x)\right) h \sum_{j=0}^{n-1} \nabla q_{j}^{*}\left(-A_{1, n} \nabla q_{n}^{*}+A_{2, n} h \sum_{j=0}^{n-1} \nabla q_{j}^{*}-2 A_{2, n} \nabla V_{n, k}^{*}\right) .
\end{aligned}
$$


Let $Q_{n}(x)$ be the last line of (139). We now estimate this function using (87), (119), (120), (126), (127) and (131)

$$
\left\|Q_{n}\right\|_{L_{2}(\Omega)} \leq 8 \bar{s}^{2} C C^{*} \sqrt{\alpha} f(\alpha)\left(C^{*}+\sqrt{\alpha} f(\alpha)+B\right) \leq \alpha^{b_{2}}, n \in[1, N] .
$$

First, we estimate $\widetilde{q}_{1,1}$. Denote $G_{1,1} \widetilde{q}_{1,1}:=\Delta \widetilde{q}_{1,1}+A_{1,1} \nabla V_{1,1} \nabla \widetilde{q}_{n, k}$. We obtain from (137)(139) that the function $\widetilde{q}_{1,1}$ satisfies boundary conditions (138) as well as the following integral identity for all functions $v \in H^{5}(\Omega)$ satisfying (138)

$$
\begin{aligned}
& \left(G_{1,1} \widetilde{q}_{1,1}, G_{1,1} v\right)+\alpha\left[\widetilde{q}_{1,1}, v\right]=\left(\widetilde{H}_{1,1}, G_{1,1} v\right)-\alpha\left[q_{1}^{*}, v\right], \\
& \widetilde{H}_{1,1}:=A_{1,1} \nabla \widetilde{V}_{1,1} \nabla q_{n}^{*}-A_{2, n} \nabla \widetilde{V}_{1,1}\left(\nabla V_{1,1}+\nabla V^{*}\right)+Q_{1} .
\end{aligned}
$$

By (108), (119) and (120) $\left\|A_{1,1} \nabla V_{1,1}\right\|_{C(\bar{\Omega})} \leq 8 B \bar{s}^{2}$. Hence, using Lemma 5.3, Theorem 5.1 and (87), we obtain

$$
\begin{aligned}
\left\|\widetilde{q}_{1,1}\right\|_{H^{5}(\Omega)} & \leq D\left(\alpha^{-1 / 2}\left\|\widetilde{H}_{1,1}\right\|_{L_{2}(\Omega)}+1\right), \\
\left\|\widetilde{q}_{1,1}\right\|_{H^{2}\left(\Omega_{\varkappa_{0}}\right)} & \leq D\left(\alpha^{-b_{1}}\left\|\widetilde{H}_{1,1}\right\|_{L_{2}(\Omega)}+\alpha^{b_{2}}\right) .
\end{aligned}
$$

Estimate now $\left\|\widetilde{H}_{1,1}\right\|_{L_{2}(\Omega)}$. By (87), (108), (120), (130), (131), (140) and (141)

$$
\left\|\widetilde{H}_{1,1}\right\|_{L_{2}(\Omega)} \leq 8 \bar{s}^{2} C^{*} B \alpha^{b_{2}}+16 \bar{s}^{2} B \alpha^{b_{2}}+\alpha^{b_{2}} \leq 8 \bar{s}^{2} B\left(C^{*}+3\right) \alpha^{b_{2}} .
$$

Assuming that

$$
D \geq 8 \bar{s}^{2} B\left(C^{*}+6\right)
$$

we obtain $\left\|\widetilde{H}_{1,1}\right\|_{L_{2}(\Omega)} \leq D \alpha^{b_{2}}$. Hence, using (142)-(144) and $b_{2}=1 / 2-b_{1}$, we obtain

$$
\begin{aligned}
\left\|\widetilde{q}_{1,1}\right\|_{H^{5}(\Omega)} & \leq D^{2}\left(\alpha^{-b_{1}}+1\right), \\
\left\|\widetilde{q}_{1,1}\right\|_{H^{2}\left(\Omega_{\varkappa_{0}}\right)} & \leq D^{2}\left(\alpha^{1 / 2-2 b_{1}}+\alpha^{b_{2}}\right) .
\end{aligned}
$$

Since $q_{1,1}=\widetilde{q}_{1,1}+q_{1}^{*}$, then by (87), (121) and (145) lead to

$$
\left\|q_{1,1}\right\|_{C^{1}(\bar{\Omega})} \leq D^{3}\left(\alpha^{-b_{1}}+2\right) \text {. }
$$

We now estimate $\left\|\widetilde{c}_{1,1}\right\|_{L_{2}\left(\Omega^{\prime}\right)}$. It follows from (46), (47) and (92)-(94) that

$$
\widetilde{c}_{1,1}=\left(-h \Delta \widetilde{q}_{1,1}+\Delta \widetilde{V}_{1,1}\right)+s_{1}^{2}\left(-h \nabla \widetilde{q}_{1,1}+\nabla \widetilde{V}_{1,1}\right)\left[-h \nabla\left(q_{11}+q_{1}^{*}\right)+\nabla\left(V_{11}+V^{*}\right)\right] .
$$

By (127) and (135)

$$
D^{2 N m+4} \alpha^{b_{2}}<N^{-1}
$$


Hence, (87), (121), (126), (144)-(147) and (149) imply that

$$
\begin{aligned}
h\left\|\Delta \widetilde{q}_{1,1}\right\|_{L_{2}\left(\Omega_{\varkappa_{0}}\right)}, h\left\|\nabla \widetilde{q}_{1,1}\right\|_{L_{2}\left(\Omega_{\varkappa_{0}}\right)} & \leq 2 D^{2} \alpha^{2 b_{2}} \leq D^{3} \alpha^{2 b_{2}} \leq \alpha^{b_{2}} N^{-1}, \\
h\left(\left\|\nabla q_{1,1}\right\|_{C(\bar{\Omega})}+2\left\|\nabla q_{1}^{*}\right\|_{C(\bar{\Omega})}\right) & \leq D^{3}\left(\alpha^{b_{2}}+4 \alpha^{1 / 2}\right) \leq N^{-1} .
\end{aligned}
$$

Next, by (131) $\left\|\nabla\left(V_{11}+V^{*}\right)\right\|_{C(\bar{\Omega})} \leq B$. Hence, using (144) and (151), we obtain

$$
s_{1}^{2}\left\|-h \nabla\left(q_{1,1}+q_{1}^{*}\right)+\nabla\left(V_{1,1}+V^{*}\right)\right\|_{C(\bar{\Omega})} \leq D \text {. Hence, (130), (144), (148) and (150) imply }
$$
that

$$
\left\|\widetilde{c}_{1,1}\right\|_{L_{2}\left(\Omega^{\prime}\right)} \leq\left\|\widetilde{c}_{1,1}\right\|_{L_{2}\left(\Omega_{\varkappa_{0}}\right)} \leq\left(B+N^{-1}\right)(D+1) \alpha^{b_{2}} \leq D^{2} \alpha^{b_{2}}
$$

Hence, (109), (124), (125), (144) and (152) lead to

$$
\left\|\nabla \widetilde{V}_{1,2}\right\|_{L_{2}(\Omega)}+\left\|\Delta \widetilde{V}_{1,2}\right\|_{L_{2}(\Omega)} \leq D^{3} \alpha^{b_{2}}
$$

We have obtained estimates (145)-(147), (150)-(153) starting from the estimates (130), (131) for functions $\widetilde{V}_{1,1}, V_{1,1}, V^{*}$. Hence, continuing this process $m$ times, using $q_{1}=q_{1, m}, c_{1}=$ $c_{1, m}$ and keeping in mind that by $(51) V_{2,1}=V_{1, m+1}$, we obtain similarly with (150)-(153)

$$
\begin{aligned}
h\left\|\nabla \widetilde{q}_{1}\right\|_{L_{2}\left(\Omega_{\varkappa_{0}}\right)}, h\left\|\Delta \widetilde{q}_{1}\right\|_{L_{2}\left(\Omega_{\varkappa_{0}}\right)} & \leq \alpha^{b_{2}} N^{-1}, \\
h\left(\left\|\nabla q_{1}\right\|_{C(\bar{\Omega})}+2\left\|\nabla q_{1}^{*}\right\|_{C(\bar{\Omega})}\right) & \leq N^{-1}, \\
\left\|c_{1}-c^{*}\right\|_{L_{2}\left(\Omega^{\prime}\right)} & \leq\left\|\widetilde{c}_{1, m}\right\|_{L_{2}\left(\Omega^{\prime}\right)} \leq D^{2 m} \alpha^{b_{2}}, \\
\left\|\nabla \widetilde{V}_{2,1}\right\|_{L_{2}(\Omega)}+\left\|\Delta \widetilde{V}_{2,1}\right\|_{L_{2}(\Omega)} & \leq D^{2 m+1} \alpha^{b_{2}} .
\end{aligned}
$$

Note that $(156)$ is $(136)$ for $n=1$. Let $\in[2, N)$. Because of (154)-(157), assume that

$$
\begin{aligned}
h \sum_{j=0}^{n-1}\left\|\nabla \widetilde{q}_{j}\right\|_{L_{2}\left(\Omega_{\varkappa_{0}}\right)}, h \sum_{j=0}^{n-1}\left\|\Delta \widetilde{q}_{j}\right\|_{L_{2}\left(\Omega_{\varkappa_{0}}\right)} & \leq \frac{n-1}{N} \alpha^{b_{2}}, \\
h \sum_{j=0}^{n-1}\left(\left\|\nabla q_{j}\right\|_{C(\bar{\Omega})}+2\left\|\nabla q_{j}^{*}\right\|_{C(\bar{\Omega})}\right) & \leq \frac{n-1}{N}, \\
\left\|\nabla \widetilde{V}_{n, 1}\right\|_{L_{2}\left(\Omega_{\varkappa_{0}}\right)}+\left\|\Delta \widetilde{V}_{n, 1}\right\|_{L_{2}\left(\Omega_{\varkappa_{0}}\right)} & \leq D^{2(n-1) m+1} \alpha^{b_{2}}:=D_{n-1} \alpha^{b_{2}}, \\
\left\|c_{n-1}-c^{*}\right\|_{L_{2}\left(\Omega^{\prime}\right)} \leq\left\|\widetilde{c}_{n-1, m}\right\|_{L_{2}\left(\Omega_{\varkappa_{0}}\right)} & \leq D^{2(n-1) m} \alpha^{b_{2}} .
\end{aligned}
$$

We are going to prove now (158)-(161) and (136) for $n:=n+1$. Because of (137), denote

$$
G_{n, 1} \widetilde{q}_{n, 1}=\Delta \widetilde{q}_{n, 1}-A_{1, n}\left(\chi_{2}(x) h \sum_{j=0}^{n-1} \nabla q_{j}-\nabla V_{n, 1}\right) \nabla \widetilde{q}_{n, 1}
$$


By (94) the function $\widetilde{q}_{n, 1}$ satisfies boundary conditions (138) as well as the following integral identity for all functions $v \in H^{5}(\Omega)$ satisfying boundary conditions (138)

$$
\left(G_{n, 1} \widetilde{q}_{n, 1}, G_{n, 1} v\right)+\alpha\left[\widetilde{q}_{n, 1}, v\right]=\left(\widetilde{H}_{n, 1}, G_{n, 1} v\right)-\alpha\left[q_{n}^{*}, v\right]
$$

Estimate the coefficient at $\nabla \widetilde{q}_{n, 1}$ in (162). Using (108), (120) and (159), we obtain

$$
\left|A_{1, n}\left(\chi_{2}(x) h \sum_{j=0}^{n-1} \nabla q_{j}-\nabla V_{n, 1}\right)\right| \leq 16 B \bar{s}^{2} .
$$

In terms of Theorem 5.1 an important feature of (164) is that this estimate is independent on $n$, implying that the same constants $D, b_{1}, b_{2}=1 / 2-b_{1}$ can be used in (165), (166) for all $n \in[2, N]$, provided that (158)-(161) are valid for $n:=n+1$. Thus, using Lemma 5.3, Theorem 5.1, (144), (163) and (164), we obtain

$$
\begin{aligned}
\left\|\widetilde{q}_{n, 1}\right\|_{H^{5}(\Omega)} & \leq D\left(\alpha^{-1 / 2}\left\|\widetilde{H}_{n, 1}\right\|_{L_{2}(\Omega)}+1\right) \\
\left\|\widetilde{q}_{n, 1}\right\|_{H^{2}\left(\Omega_{\varkappa_{0}}\right)} & \leq D\left(\alpha^{-b_{1}}\left\|\widetilde{H}_{n, 1}\right\|_{L_{2}(\Omega)}+\alpha^{b_{2}}\right) .
\end{aligned}
$$

Hence, using (85), (120), (121), (139), (140), (144), (158)-(161), Theorem 6.1 and that $B>2$, we obtain

$$
\begin{aligned}
\left\|\widetilde{H}_{n, 1}\right\|_{L_{2}(\Omega)} \leq & 8 \bar{s}^{2}\left(\frac{n-1}{N} \alpha^{b_{2}}+D_{n-1} \alpha^{b_{2}}\right) C^{*}+8 \bar{s}^{2} \frac{n-1}{N} \alpha^{b_{2}}\left(\frac{n-1}{N}+2 B\right) \\
& +8 \bar{s}^{2} D_{n-1} \alpha^{b_{2}}\left(\frac{2(n-1)}{N}+2 B\right)+\alpha^{b_{2}} \\
\leq & 8 \bar{s}^{2} D_{n-1} \alpha^{b_{2}}\left(3+4 B+3 C^{*} / 2\right) \leq D D_{n-1} \alpha^{b_{2}} .
\end{aligned}
$$

Hence, (121), (149), (160), (165) and (166) imply that

$$
\begin{aligned}
& h\left(\left\|\nabla q_{n, 1}\right\|_{C(\bar{\Omega})}+2\left\|\nabla q_{n, 1}^{*}\right\|_{C(\bar{\Omega})}\right) \leq D^{3} D_{n-1} \alpha^{b_{2}} \leq D^{2 N m+4} \alpha^{b_{2}} \leq N^{-1}, \\
& h\left\|\widetilde{q}_{n, 1}\right\|_{H^{2}\left(\Omega_{\varkappa_{0}}\right)} \leq D\left(D D_{n-1} \alpha^{2 b_{2}}+\alpha^{b_{2}+1 / 2}\right) \leq D^{2 N m+4} \alpha^{2 b_{2}} \leq \alpha^{b_{2}} N^{-1} .
\end{aligned}
$$

We obtain similarly with (148)

$$
\begin{array}{r}
\widetilde{c}_{n, 1}=\left(-h \Delta \widetilde{q}_{n, 1}-h \sum_{j=0}^{n-1} \Delta \widetilde{q}_{j}+\Delta \widetilde{V}_{n, 1}\right)+s_{n}^{2}\left(-h \nabla \widetilde{q}_{n, 1}-h \sum_{j=0}^{n-1} \nabla \widetilde{q}_{j}+\nabla \widetilde{V}_{n, 1}\right) . \\
\cdot\left[-h \nabla\left(q_{n, 1}+q_{n}^{*}\right)-h \sum_{j=0}^{n-1} \nabla\left(q_{j}+q_{j}^{*}\right)+\nabla\left(V_{n, 1}+V^{*}\right)\right] .
\end{array}
$$


Hence, using (144), (158)-(160), (167) and (168), we obtain

$$
\left\|\widetilde{c}_{n, 1}\right\|_{L_{2}\left(\Omega_{\varkappa_{0}}\right)} \leq\left(\frac{n}{N} \alpha^{b_{2}}+D_{n-1} \alpha^{b_{2}}\right)\left[1+\bar{s}^{2}\left(\frac{n}{N}+B\right)\right] \leq D D_{n-1} \alpha^{b_{2}} .
$$

Hence, (109), (124) and (125) imply that

$$
\left\|\nabla \widetilde{V}_{n, 2}\right\|_{L_{2}(\Omega)}+\left\|\Delta \widetilde{V}_{n, 2}\right\|_{L_{2}(\Omega)} \leq D^{2} D_{n-1} \alpha^{b_{2}} .
$$

Similarly for $k=1, \ldots, m$

$$
\left\|\widetilde{c}_{n, k}\right\|_{L_{2}\left(\Omega_{\varkappa_{0}}\right)} \leq D^{2 k-1} D_{n-1} \alpha^{b_{2}},\left\|\nabla \widetilde{V}_{n, k+1}\right\|_{L_{2}(\Omega)}+\left\|\Delta \widetilde{V}_{n, k+1}\right\|_{L_{2}(\Omega)} \leq D^{2 k} D_{n-1} \alpha^{b_{2}} .
$$

Hence, similarly with the above, we obtain that estimates (167), (168) are valid for functions $q_{n, k}, \widetilde{q}_{n, k}$. This implies the validity of (158) and (159) for $n:=n+1$. Similarly

$$
\begin{gathered}
\left\|c_{n, k}-c^{*}\right\|_{L_{2}\left(\Omega^{\prime}\right)} \leq\left\|\widetilde{c}_{n, k}\right\|_{L_{2}\left(\Omega_{\varkappa_{0}}\right)} \leq D^{2 k} D_{n-1} \alpha^{b_{2}}=D^{2(n-1) m+2 k} \alpha^{b_{2}}, k \in[1, m], \\
\left\|\nabla \widetilde{V}_{n, m+1}\right\|_{L_{2}(\Omega)}+\left\|\Delta \widetilde{V}_{n, m}\right\|_{L_{2}(\Omega)} \leq D^{2 m} D_{n-1} \alpha^{b_{2}}=D^{2 n m+1} \alpha^{b_{2}} .
\end{gathered}
$$

The last two estimates establish (160) and (161) for $n:=n+1$.

\section{Numerical Studies}

\subsection{Main discrepancies between the convergence analysis and the numerical implementation}

\subsubsection{Discussion of the topic of discrepancies}

As to the general philosophy of numerical solutions of challenging nonlinear problems, both well-posed and ill-posed ones, it is well known that some discrepancies between the convergence analysis and numerical implementations are almost inevitable. The main reason is that because of the complicated structure of those problems, the theory usually can grasp only a part of numerical studies rather than all aspects. In general, given a complicated nonlinear ill-posed problem, it is almost impossible to obtain accurate computational results if following the theory exactly. Thus, it is well known that computations are usually carried out under less restrictive conditions than ones imposed by the convergence analysis.

For example, as a rule, constants in convergence theorems are significantly over/ underestimated (maybe with the only exception of a few very simple linear problems). In addition, the theory works for a continuous case meaning an infinitely dimensional space. On the other hand, in real computations one always works with a discrete case of the FEM/FDM in a finite dimensional functional space. Furthermore, in the case of an ill-posed problem the minimal spatial grid size $h_{s p}$ of the FEM/FDM often serves as an implicit regularization parameter 
for the discrete case (naturally $h_{s p}$ is different from the above step size $h$ in the $s$-direction). Thus, $h_{s p}$ cannot be taken too small: otherwise that functional space would effectively become an infinitely dimensional one. For example, in our numerical testing below we have used $h_{s p}=0.125$ to minimize the QRM functional (56) via the FDM. However, our attempt to decrease it to $h_{s p}=0.0625$ has significantly worsened results. The bound from below for $h_{s p}$ imposes an upper bound on the dimension of the corresponding finite dimensional functional space. Thus, already over/under-estimated constants of convergence theorems for the continuous case become even more over/under-estimated for the finite dimensional case with an upper bound on its dimension.

For example, Theorem 7.1 imposes smallness conditions on the parameter $\alpha$ as well as on other parameters in (125)-(127), which is an "ideal limiting case" for our problem. We note that a similar situation quite often occurs when one proves convergence theorems about nonlinear problems, both well-posed and ill-posed ones, assuming that some parameters are "sufficiently small". Despite this assumption, it is often the case in real computations that those parameters are not too small. Another example is an accurate imaging from experimental data with a large noisy component in $[8,27]$ despite the fact that the convergence analysis of $[4,5]$ requires a sufficiently small error in the input data. Figures 3-5 in [27] as well as Figure 2-a in [8] give an idea about the amount of the noise in those data.

Having stated the above, we nevertheless point out that the convergence analysis is obviously a very important element of numerical studies. This analysis provides an analytical guidance for computations, qualitatively explains numerical results and ensures that at least in an "ideal limiting case" the convergence is guaranteed. In addition, regardless on some deviations, numerical implementations are usually guided by convergence theorems. Thus, a certain reasonable balance between the convergence analysis and the numerical implementation should take place.

In particular, speaking about our problem, we believe that various conditions in our convergence analysis might be relaxed if working with a discrete case with a lower bound imposed on $h_{s p}, h_{s p} \geq h_{0}=$ const $>0$. To analyze the QRM in this case, one needs to apply a discrete version of the Carleman estimate of Lemma 5.1, see, e.g. [13, 25] for discrete Carleman estimates for elliptic operators. The latter, in turn should likely result in relaxed conditions of a discrete analog of Theorem 7.1. This will be one of topics of our future research.

\subsubsection{Main discrepancies}

The first main discrepancy is with regard to Lemma 3.1 about an easily verifiable sufficient condition of the regularity of geodesic lines. In general, such a condition is unknown, except of the trivial case when the function $c(x)$ is close to a constant. On the other hand, the authors are unaware about any reasonable results for CIPs for hyperbolic PDEs without either the assumption of the regularity of geodesic lines or a somewhat close assumption. We verify the asymptotic behavior (36) computationally, see subsection 7.2 of [4].

The second main discrepancy is that we replace in our computations $\alpha\|u\|_{H^{5}(\Omega)}^{2}$ in (56) 
with $\alpha\|u\|_{H^{2}(\Omega)}^{2}$, because the latter is simpler to implement numerically. One of the reasons why this works computationally might be because of the above arguments about finite dimensional spaces, since all norms are equivalent in such spaces.

The third main discrepancy is that we conduct computations for the case when the point source in (2) is replaced with the plane wave. This is because our current software works with the plane wave only and it would take a time consuming effort to make it work for the point source. In addition, the case of the plane wave is reasonable for our target application to imaging of plastic land mines, since the wave radiated by a point source effectively becomes a plane wave when that source is located far from the domain of interest. We have chosen the point source in (2) with the goal to use Lemma 3.1 which actually can be derived from the construction of the fundamental solution of a general hyperbolic equation in Theorem 4.1 of [38]. Other than this, the above technique can be extended to the case of the plane wave and this might be one of topics of our future research.

When this manuscript was ready for submission, M.V. Klibanov and N. Pantong (a coauthor of [30]) have implemented the case of the point source. Computational results were almost the same as ones below. However, keeping in mind considerations of brevity as well as the necessity to submit a separate paper with Pantong as a coauthor, the authors have decided not to present the point source case here.

The fourth main discrepancy is that we conduct computations for the 2-D case since this case is both easier to implement numerically. Another important factor is that the 2-D case is faster to compute. The 3-D case is quite feasible [30]. Indeed, the 3-D image in [30] was computed from the forward problem data simulated in 3-D. At the same time, twenty four 2-D inverse problems in corresponding 2-D cross-sections were solved simultaneously on twenty four processors and the 3-D image was formed this way.

\subsection{A simplified model of imaging of plastic land mines and some details of the numerical implementation}

We outline both topics of this subsection only briefly here referring to [30] for details. In the spirit of sub-subsection 8.1.1, we have made some simplifications in our mathematical model. The first main simplification of our model is that we consider the 2-D instead of the $3-\mathrm{D}$ case. Second, we ignore the air/ground interface, assuming that the governing PDE is valid on the entire 2-D plane. Indeed, our experience of working with the experimental data of [31] has indicated that the reflection from the air/ground interface can be removed from the data via a new data pre-processing procedure. The idea of a similar data pre-processing procedure can be found in $[8,27]$. Let the ground be $\{\mathbf{x}=(x, z): z>0\} \subset \mathbb{R}^{2}$. Suppose that a polarized electric field is generated by a plane wave, which is initialized at the line $\left\{z=z^{0}<0, x \in \mathbb{R}\right\}$ at the moment of time $t=0$. As it was pointed out in section 2 , the following hyperbolic equation can be derived from the Maxwell equations

$$
\begin{aligned}
\varepsilon_{r}(\mathbf{x}) u_{t t} & =\Delta u,(\mathbf{x}, t) \in \mathbb{R}^{2} \times(0, \infty) \\
u(\mathbf{x}, 0) & =0, u_{t}(\mathbf{x}, 0)=\delta\left(z-z^{0}\right)
\end{aligned}
$$


where the function $u(\mathbf{x}, t)$ is a component of the electric field and $\varepsilon_{r}(\mathbf{x})$ is the spatially distributed dielectric constant. We assume that the function $\varepsilon_{r}(\mathbf{x})$ satisfies conditions (3), (4) (in 2-D). Let the function $w_{0}(z, s)=\exp \left(-s\left|z-z_{0}\right|\right) /(2 s)$ be the one which correspond to the Laplace transform (9) of the incident plane wave with $\varepsilon_{r}(\mathbf{x}) \equiv 1$. Applying (9) to (169), we obtain the following analog of the problem (11), (12)

$$
\begin{aligned}
\Delta w-s^{2} \varepsilon_{r}(\mathbf{x}) w & =-\delta\left(z-z^{0}\right), s \geq \underline{s}^{0}=\text { const. }>0, \mathbf{x} \in \mathbb{R}^{2}, \\
\lim _{|\mathbf{x}| \rightarrow \infty}\left(w-w_{0}\right)(\mathbf{x}, s) & =0 .
\end{aligned}
$$

It is well known that the maximal depth of an antipersonnel land mine does not exceed about $10 \mathrm{~cm}=0.1 \mathrm{~m}$. So, we model these mines as small squares with the $0.1 \mathrm{~m}$ length of sides, and their centers are at the depth of $0.1 \mathrm{~m}$. We set

$\widetilde{\Omega}=\{\mathbf{x}=(x, z) \in(-0.30,0.30) m \times(0,0.6) m\}$. Introducing dimensionless spatial variables $\mathbf{x}^{\prime}=\mathbf{x} /(0.1 \mathrm{~m})$ without changing notations, we obtain $\Omega=(-3,3) \times(0,6)$, sides of small squares modeling mines equal 1 and depths of their centers are at $\{z=1\}$. We took $\Omega_{P_{0}}=(-3,3) \times(0,3)$ (subsection 4.1). Tables of dielectric constants [39] show that $\varepsilon_{r}=5$ and $\varepsilon_{r}=22$ in the dry sand in the trinitrotoluene (TNT) respectively, meaning $22 / 5=4.4$ of the mine/background contrast. Hence, considering new parameters $\varepsilon_{r}^{\prime}=\varepsilon_{r} / 5, s^{\prime}=s \cdot 0.1 \cdot \sqrt{5}$ and not changing notations, we obtain

$$
\varepsilon_{r}(\operatorname{dry} \text { sand })=1, \varepsilon_{r}(\mathrm{TNT})=4.4 .
$$

Because of (173) and (3), we impose $\varepsilon_{r}(\mathbf{x}) \in[1,8], \varepsilon_{r}(\mathbf{x})=1$ outside of the rectangle $\Omega_{P_{0}}$. We have modified our algorithm of section 4 via considering functions $\widehat{v}(x, s)=$ $s^{-2} \ln \left(w / w_{0}\right), \widehat{q}(x, s)=\partial_{s} \widehat{v}(x, s)$ instead of $v(x, s)=s^{-2} \ln (w), q(x, s)=\partial_{s} v(x, s)$. This has resulted in obvious modifications of above equations. A slight modification of Theorem 7.1 can be proven for this case. We have observed in our computations that at the measurement side $\Gamma_{1}=\{x \in(-3,3), z=0\}$ of the above square $\Omega$ the ratio $\left(w / w_{0}\right)(x, 0, s) \approx 1$ for $s>1.2$ which means a poor sensitivity of $\Gamma_{1}$ to the presence of abnormalities for values of the pseudo frequency $s>1.2$. The best sensitivity was for $s \in(0.5,1.2)$. Hence, one should expect that the modified tail function $\widehat{V}(\mathbf{x}, s)=V(\mathbf{x}, s)-s^{-2} \ln w(\mathbf{x}, s) \approx 0$ for $s>1.2$ at least for $\mathbf{x}$ close to $\Gamma_{1}$. Hence, we have chosen $\bar{s}=1.2$ and $s \in[0.5,1.2]:=[\underline{s}, \bar{s}]$. However, if we would work in the original domain $\widetilde{\Omega}$ making spatial variables dimensionless as $\mathbf{x}^{\prime \prime}=\mathbf{x} /(1 m)$, then $s^{\prime \prime}=\sqrt{5} s$ implying that $\bar{s}^{\prime \prime}=12$, which can be considered as a large pseudo frequency. The latter shows that in practical computations the above notion of sufficiently large $\bar{s}$ is actually a conditional one and depends on particular ranges of parameters at hands (in fact, this is in the spirit of subsection 8.1).

To simulate the data at $\{z=0\}$ for the inverse problem, we have solved the problem (171), (172) via the FDM for a number of points $s_{n} \in[0.5,1.2]$. For comparison, we have also solved once the problem (169), (170) and have applied the Laplace transform (9) then. Imaging results were almost the same. We have added the random noise of the $5 \%$ level to the simulated Dirichlet data at $\Gamma_{1}$ via $w_{\sigma}\left(x_{i}, 0, s_{n}\right)=w\left(x_{i}, 0, s_{n}\right)\left(1+\sigma \omega_{n}\right), \sigma=0.05$, where $\left\{x_{i}\right\}$ are grid points of the FDM for the forward problem solution and $\omega \in(-1,1)$ 
is a random variable. To calculate the derivative $\partial_{s}\left[s^{-2} \ln \left(w_{\sigma} / w_{0}\right)\left(x_{i}, 0, s_{n}\right)\right]$ (to obtain the boundary data for $\left.\widehat{q}\left(x_{i}, s_{n}\right)\right)$, we have smoothed first values of $w_{\sigma}\left(x_{i}, 0, s_{n}\right)$ with respect to $s$ via cubic $B$-splines similarly with, e.g. [22]. Next, we have used finite differences to calculate the desired derivative.

The modified functional (56) was written in the FDM form. Its minimization was performed with respect to values of the function $u$ at grid points via the conjugate gradient method. We have chosen $\alpha=0.04$ and the spatial grid step size $h_{s p}=0.125$. First, we have solved the problem (104), (105) via the QRM and thus have calculated the first tail $V_{1,1}(x)$ in (107). Next, we have continued as in section 4 with $m:=10$. The above algorithm has provided us with the function $\varepsilon_{r, g l o b}(\mathbf{x})$ (Definition 6.1).

We have observed in our computations that the above algorithm can accurately image locations of mine-like targets. However, values of the function $\varepsilon_{r, g l o b}(\mathbf{x})$ near points of local maxima were not imaged accurately. Thus, similarly with $[5,6,7,8,30]$, we have applied a two-stage numerical procedure, see comments in subsection 1.1. While the first stage was the one described above, on the second stage we have minimized the Tikhonov regularization functional via the gradient method taking the function $\varepsilon_{r, g l o b}(\mathbf{x})$ as the starting point. Since the second stage is not a focus of the current paper, we refer to [30] for a detailed description. Our attempt to use the gradient method without the first stage did not lead to accurate results. The latter indicates the importance of the first stage.

\subsection{Numerical results}

In this subsection we present two numerical examples of the performance of our numerical method for computationally simulated data using the above mathematical model of imaging of plastic antipersonnel land mines.

Test 1 . We test our numerical method for the case when plastic land mines are modeled by two small squares with the same size $s z=1$ of their sides. Also, $\varepsilon_{r}=4.4$ in both of them and $\varepsilon_{r}=1$ everywhere else, see (173) and Figure 8.1-a. Centers of these squares are at $\left(x_{1}^{*}, z_{1}^{*}\right)=(-1.5,1.0)$ and $\left(x_{2}^{*}, z_{2}^{*}\right)=(1.5,1)$. However, we do not assume a priori in our algorithm neither the presence of these squares nor a knowledge of $\varepsilon_{r}(\mathbf{x})$ at any point of the rectangle $\Omega$. Figure 8.1-b shows the image of $\varepsilon_{r, g l o b}(\mathbf{x})$. Locations of both local maxima are imaged well. However, values of those maxima are 2.8 , which is about $36 \%$ off the correct one. Figure 8.1-c displays the final image after applying the second stage of our two-stage numerical procedure. Locations of both targets are imaged well. In addition, maximal values of the computed coefficient $\varepsilon_{r, \text { final }}$ in both targets are $\max \left(\varepsilon_{r, \text { final }}\right)=4.4$, which is accurate. Likewise, $\varepsilon_{r, \text { final }}(\mathbf{x})=1$ outside of those spots, which is also accurate.

Test 2. We test our numerical method for the case of the same two squares as ones above. Now, however we have $\varepsilon_{r}=6$ in the left square, $\varepsilon_{r}=4$ in the right square and $\varepsilon_{r}=1$ everywhere else, see (173). Again, we have obtained accurate images, see Figure 8.2 and the legend for it.

Although mine-like targets are symmetrically located in both tests, values of the function $\varepsilon_{r}(\mathbf{x})$ are not symmetric in Test 2. A similar image quality for the case of asymmetrical 
locations of two targets was obtained in [30].

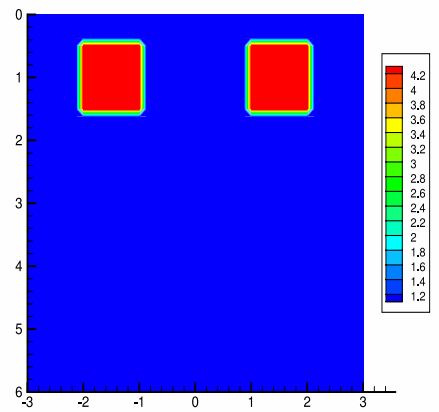

a)

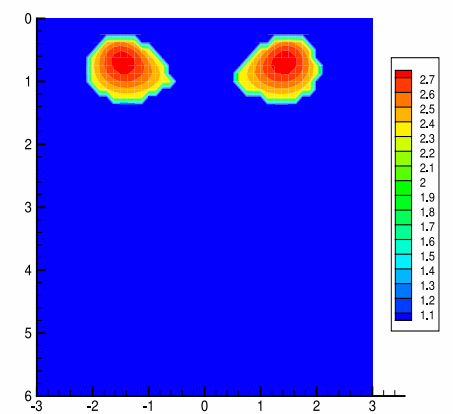

b)

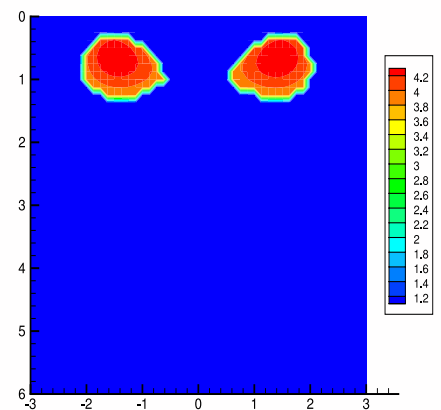

c)

Figure 1: Test 1. The plane wave falls from the top and the data are collected on the top boundary. a) Correct image of two mine-like targets, $\varepsilon_{r}=4.4$ in both small squares and $\varepsilon_{r}=1$ everywhere else, see (173). b) The image of the function $\varepsilon_{r, g l o b}(\mathbf{x})$. While locations of targets are imaged accurately, values of local maxima are 2.8, which is $36 \%$ off the correct one. c) The final image of the function $\varepsilon_{r, \text { final }}(\mathbf{x})$ after applying the second stage of the two-stage numerical procedure. Locations of both mine-like targets are accurately imaged. Likewise, $\max \left(\varepsilon_{r, f i n a l}\right)=4.4$ in both spots and $\varepsilon_{r, \text { final }}=1$ outside of these spots, which is also accurate.

Acknowledgments. This work was supported by the U.S. Army Research Laboratory and U.S. Army Research Office under the grant number W911NF-08-1-0470, by Visby Program of Swedish Institute and by the Russian Foundation for Basic Research under the grant number 08-01-00312.

\section{References}

[1] N.V. Alexeenko, V.A. Burov and O.D. Rumyantseva, Solution of threedimensional acoustical inverse problem: II. Modified Novikov algorithm, Acoustical Physics, 54 (2008), pp. 407-419.

[2] A.B. Bakushinsky And M.Yu. Kokurin, Iterative Methods for Approximate Solutions of Inverse Problems, Springer, Dordrecht, 2004.

[3] H. Bateman And A. ERdelyi, Tables of Integral Transforms, Vol. 1, McGrawHill, New York, 1954.

[4] L. Beilina And M. V. Klibanov, A globally convergent numerical method for a coefficient inverse problem, SIAM J. Sci. Comp., 31 (2008), pp. 478-509. 


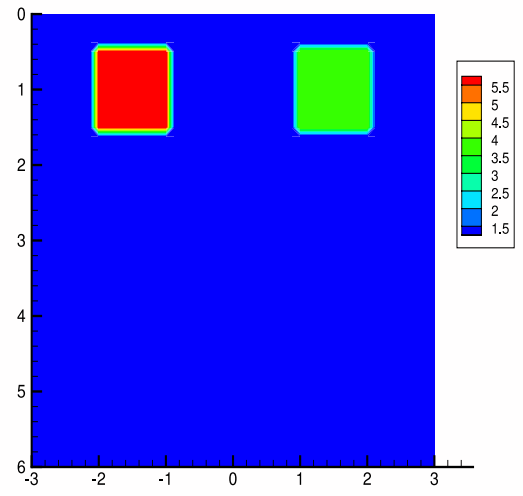

a)

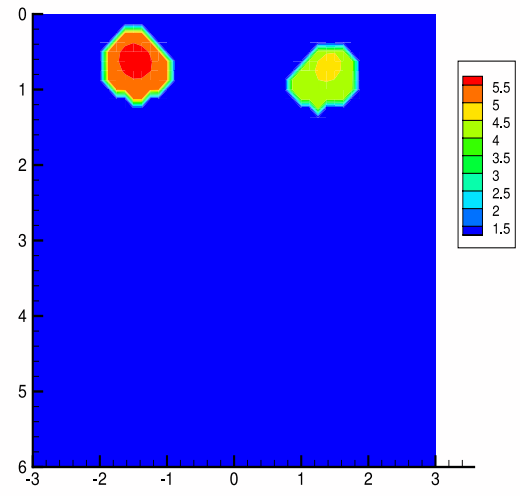

b)

Figure 2: Test 2. The plane wave falls from the top and the data are collected on the top boundary. a) Correct image of two mine-like targets, $\varepsilon_{r}=6$ in the left small square, $\varepsilon_{r}=4$ in the right small square and $\varepsilon_{r}=1$ everywhere else, see (173). b) The final image of $\varepsilon_{r, \text { final }}(\mathbf{x})$ obtained after applying the two-stage numerical procedure. Locations of both mine-like targets are imaged accurately. In addition, $\max \left(\varepsilon_{r, \text { final }}\right)=6$ and $\max \left(\varepsilon_{r, \text { final }}\right)=4.5$ in the left and right spots respectively, and $\varepsilon_{r, \text { final }}(\mathbf{x})=1$ outside of these spots.

[5] L. Beilina And M. V. Klibanov, Synthesis of global convergence and adaptivity for a hyperbolic coefficient inverse problem in 3D, J. Inverse and Ill-Posed Problems, 18 (2010), pp. 85-132.

[6] L. Beilina And M. V. Klibanov, A posteriori error estimates for the adaptivity technique for the Tikhonov functional and global convergence for a coefficient inverse problem, Inverse Problems, 26 (2010), 045012.

[7] L. Beilina, M. V. Klibanov and M.Yu. Kokurin, Adaptivity with relaxation for ill-posed problems and global convergence for a coefficient inverse problem, J. Mathematical Sciences, 167 (2010), pp. 279-325.

[8] L. Beilina And M.V. Klibanov, Reconstruction of dielectrics from experimental data via a hybrid globally convergent/adaptive algorithm, Inverse Problems, 26 (2010), 125009.

[9] M.I. Belishev, Wave bases in multidimensional inverse problems, Math. USSR Sb. 67 (1990), 23-42.

[10] M.I. Belishev And V.Y. Gotlib, Dynamical variant of the bc method and numerical testing, J. Inverse and Ill-Posed Problems, 7 (1999), 221-240. 
[11] J. Bikowski, K. Knudsen And J.L. Mueller, Direct numerical reconstruction of conductivities in three dimensions using scattering transforms, Inverse Problems, 27, (2011), 015002 .

[12] M. Born And E. Wolf, Principles of Optics : Electromagnetic Theory of Propagation, Interference and Diffraction of Light, Cambridge University Press, Cambridge, 1999.

[13] F. Boyer, F. Hubert and J. Le Rousseau, Discrete Carleman estimates for elliptic operators in arbitrary dimension and applications, SIAM J. Control Optim., 48 (2010), pp. 5357-5397.

[14] L. Bourgeois And J. DARDe, About stability and regularization of ill-posed elliptic Cauchy problems: the case of Lipschitz domains, Applicable Analysis, 89 (2010), pp. 1745-1768.

[15] L. Bourgeois And J. DARDe, A duality-based method of quasi-reversibility to solve the Cauchy problem in the presence of noisy data, Inverse Problems, 26 (2010), 095016.

[16] H. Cao, M.V. Klibanov and S.V. Pereverzev, A Carleman estimate and the balancing principle in the quasi-reversibility method for solving the Cauchy problem for the Laplace equation, Inverse Problems, 25 (2009), 35005.

[17] M. Cheney And D. IsAacson, Inverse problems for a perturbed dissipative half-space, Inverse Problems, 11 (1995), pp. 865-888.

[18] C. Clason and M.V. Klibanov, The quasi-reversibility method for thermoacoustic tomography in a heterogeneous medium, SIAM J. Sci. Comp., 30 (2007), pp. 1-23.

[19] H.W. Engl, M. Hanke And A. Neubauer, Regularization of Inverse Problems, Kluwer Academic Publishers, Boston, 2000.

[20] A. Friedman, Partial Differential Equations of Parabolic Type, Prentice Hall, Inc., Englewood Cliffs, N.J., 1964.

[21] D. Gilbarg and N.S. Trudinger, Elliptic Partial Differential Equations of Second Order, Springer-Verlag, Berlin, 1983.

[22] Yu. A. Grayzin, M.V. Klibanov and T.R. Lucas, Numerical solution of a subsurface inverse imaging problem, SIAM J. Appl. Math., 62 (2001), pp. 664-683.

[23] S.I. Kabanikhin, A.D. Satybaev and M.A. Shishlenin, Direct Methods of Solving Multidimensional Inverse Hyperbolic Problems, VSP, Utrecht, The Netherlands, 2004.

[24] B. Kaltenbacher, A. Neubauer and O. Scherzer, Iterative Regularization Methods for Nonlinear Ill-Posed Problems, de Gruiter, New York, 2008. 
[25] M.V. Klibanov and F. Santosa, A computational quasi-reversibility method for Cauchy problems for Laplace's equation, SIAM J. Appl. Math., 51 (1991), pp. 16531675.

[26] M.V. Klibanov, Inverse problems and Carleman estimates, Inverse Problems, 8 (1992), pp. 575-596.

[27] M.V. Klibanov, M.A Fiddy, L. Beilina, N. Pantong and J. Schenk, Picosecond scale experimental verification of a globally convergent algorithm for a coefficient inverse problem, Inverse Problems, 26 (2010), 045003.

[28] M.V. Klibanov And A. Timonov, Carleman Estimates for Coefficient Inverse Problems and Numerical Applications, VPS, The Netherlands, 2004.

[29] A.V. Kuzhuget and M.V. Klibanov, Global convergence for a 1-D inverse problem with application to imaging of land mines, Applicable Analysis, 89 (2010), pp. 125-157.

[30] A.V. Kuzhuget, N. Pantong and M.V. Klibanov, A globally convergent numerical method for a coefficient inverse problem with backscattering data, Proceedings of the International Conference in Inverse Problems 2010 (Hong Kong), to be published in Methods and Applications of Analysis; also available on-line at http://www.ma.utexas.edu/mp_arc/index-10.html.

[31] A.V. Kuzhuget, M.V. Klibanov, A. Sullivan, L. Nguyen and M.A. Fiddy, Blind experimental data for an approximately globally convergent algorithm for an inverse problem, in preparation.

[32] O.A. Ladyzhenskaya, V.A. Solonnikov and N.N. Uralceva, Linear and Quasilinear Equations Of Parabolic Type, AMS, Providence: RI, 1968.

[33] R. Lattes and J.-L. Lions, The Method of Quasireversibility: Applications to Partial Differential Equations, Elsevier, New York, 1969.

[34] M.M. Lavrentiev, V.G. Romanov and S.P. Shishatskit, Ill-Posed Problems of Mathematical Physics and Analysis, AMS, Providence, RI, 1986.

[35] V.P. Mikhailov, Partial Differential Equations, Mir Publishers, Moscow (in English), 1978.

[36] L. Nguyen, D. Wong, M. Ressler, F. Koenig, B. Stanton, G. Smith, J. SICHINA AND K. KAPPRA, Obstacle avolidance and concealed target detection using the Army Research Lab ultra-wideband synchronous impulse Reconstruction (UWB SIRE) forward imaging radar, Proc. SPIE, 6553 (2007), pp. 65530H (1)-65530H (8).

[37] R.G. Novikov, The $\partial$-bar method to approximate inverse scattering at fixed energy in three dimensions, Int. Math. Research Papers, 6 (2005), 287-349. 
[38] V.G. Romanov, Inverse Problems of Mathematical Physics, VNU Press, Utrecht, The Netherlands, 1986.

[39] Tables of dielectric constants at http://www.asiinstr.com/technical/Dielectric\%20Constants.htm

[40] A.N. Tikhonov, A.V. Goncharsky, V.V. Stepanov and A.G. Yagola, $N u$ merical Methods for Solutions of Ill-Posed Problems, Kluwer, London, 1995. 